\title{
ON RESOLVENT OF MULTI-DIMENSIONAL OPERATORS WITH FREQUENT ALTERNATION OF BOUNDARY CONDITIONS: CRITICAL CASE
}

\author{
T.F. SHARAPOV
}

\begin{abstract}
We consider an elliptic operator in a multi-dimensional domain with frequent alternation of Dirichlet and Robin conditions. We study the case, when the homogenized operator has Robin condition with an additional coefficient generated by the geometry of the alternation. We prove the norm resolvent convergence of the perturbed operator to the homogenized one and obtain the estimate for the convergence rate. We construct the complete asymptotic expansion for the resolvent in the case, when it acts on sufficiently smooth functions.
\end{abstract}

Keywords: frequent alternation, homogenization, norm resolvent convergence, asymptotics

Mathematics Subject Classification: 35P15, 35C20, 35B25

\section{INTRODUCTION}

Elliptic boundary value problems with frequent alternation of boundary conditions arise in various applications. Let us describe briefly the formulation of such boundary value problems. On the boundary of a domain one chooses a set consisting of many disjoint pieces. This set depends on one or several small parameters. The measure of each part and the distances between neighbouring components tend to zero as these small parameter tend to zero, while the total amount of the pieces in the chosen set increases unboundedly. On these sets we impose Dirichlet condition, while on the other part of the boundary is subject to Neumann or Robin condition. There were considered also the cases, when the described alternation of the boundary conditions was imposed not on the whole boundary but only on its fixed part. The rest of the boundary was subject to one of the classical condition.

The homogenization of the elliptic boundary value problems in domains with frequent alternation of boundary conditions were considered in many works [1]-[14]. Most part of them were devoted to the case of bounded domains with a sufficiently smooth boundary. The main results of these works were the determination of the homogenized (limiting) problem and the proof of the convergence theorems for their solutions. The homogenized problems were the problems for the same equations in the same domains but subject to one of the classical boundary condition instead of the frequent alternation. It was shown in these works that the form of the homogenized operator, namely, the boundary condition depends on the ratio between the measures of parts of the boundary with different types of boundary conditions. The most part of the results on the convergence of the solutions were proved in the sense of the weak or

T.F. SHARAPOV, On RESOLVENT OF MULTI-DIMENSIONAL OPERATORS WITH FREQUENT ALTERNATION OF BOUNDARY CONDITIONS: CRITICAL CASE.

(C) Sharapov T.F. 2016.

The study is partially financially supported by RFBR (grant no. 15-31-20037-mol_ved_a (the results on resolvent convergence) and RSF 14-11-00078 (construction of the asymptotics for the resolvent).

Submitted March 28, 2016. 
strong resolvent convergence. Namely, the solution to the perturbed problem converges to the solution of the homogenized one weakly or strongly in $W_{2}^{1}$ or strongly in $L_{2}$. In [1], [6], for a bounded domain with a periodic alternation of Dirichlet and Neumann or Robin conditions, homogenized problems were described. The convergence in a non-periodic case was studied in [3], 4]. Apart from determining the form of the homogenized problems, in some cases the estimates for the convergence rate were proved. The estimates for the convergence rate $f$ a periodic alternation of boundary conditions were obtained in [1], [10]. Similar estimates for a non-periodic alternation were established in [3], 7], 8], [9], 13], 14].

One more type of the convergence is the norm resolvent convergence. In [15]-[20] there were considered elliptic operators in an infinite straight planar strip with a frequent alternation of boundary conditions. The norm resolvent convergence was proved for all possible homogenized operators as well as for periodic and non-periodic alternations. The estimates for the rate of convergence were obtained. Similar results with a periodic alternation of boundary conditions were established in [21], [22]. In [13], [14], an elliptic operator was considered in a multidimensional unbounded domain with a non-periodic alternation of boundary conditions. The norm resolvent convergence was proved and the sharp order estimates for the convergence rate were obtained.

There are many works in which the asymptotics for solutions of problems with frequent alternation of boundary conditions were constructed (see, for instance, [9], [13], [14], [17], [18], [23]-27]). In [23], [24], [26] there were constructed the asymptotics for two-dimensional problems with a periodic alternation of Dirichlet and Neumann conditions, while in [9] the same was done for a non-periodic alternation of Dirichlet and Robin conditions. Similar results were obtained in work [27] for a three-dimensional cylinder with a frequent alternation of Dirichlet and Neumann conditions on thin strips located on the lateral surface. In work [25], the boundary value problem for Poisson equation was considered in a multi-dimensional layer bounded by two hyperplanes, the solution was assumed to be periodic. A complete asymptotic expansion was obtained for the solution of the considered problem. In [13, [14] there were considered boundary value problems for a second order elliptic equation in an unbounded multi-dimensional domain with frequent alternation of Dirichlet and Robin conditions. For the solutions of the considered problems the complete asymptotic expansion was constructed in [13] in the case of the homogenized Dirichlet condition, while in [14] the complete two-parametric asymptotic expansion was obtained.

In the present work we consider an elliptic operator in an arbitrary unbounded multidimensional domain with a non-periodic alternation of boundary conditions. We also consider the case, when the domain is bounded. We study the case of alternating Dirichlet and Robin conditions. The alternation is imposed on the whole boundary or on its fixed part. In the latter case on the rest of the boundary we impose Robin condition. In the problem we choose two character small parameters describing the sizes of the Dirichlet and Robin parts of the boundary. As these parameters tend to zero, the amount of the Dirichlet parts increases unboundedly, while the measure of each part and the distances between them tend to zero. We consider the case, when the homogenized operator involves the Robin condition with an additional coefficient generated by the geometry of the alternation. We study the behavior of the resolvent of the perturbed operator when the mentioned small parameter tend to zero. The first main result result is the proof of the norm resolvent convergence of the perturbed operator to the homogenized one in the sense of norm of operator acting in $L_{2}$; the estimates for the convergence rates are also obtained. Nevertheless, we show that by employing a special boundary corrector we can obtain the convergence of the perturbed operator to the homogenized one in the sense of the norm of an operator acting from $L_{2}$ into $W_{2}^{1}$. The second main result is the complete asymptotic expansion for the resolvent in an unbounded domain with an additional 
assumption that the alternation of boundary conditions has a periodic structure and is imposed on the hyperplane, while the resolvent acts on sufficiently smooth functions.

\section{Formulation of the Problem}

Let $x=\left(x^{\prime}, x_{n}\right), x^{\prime}=\left(x_{1}, x_{2}, \ldots, x_{n-1}\right)$ be Cartesian coordinates in $\mathbb{R}^{n}$ and $\mathbb{R}^{n-1}$, respectively, $\Omega$ be an arbitrary domain $\mathbb{R}^{n}, n \geqslant 3$ with a boundary in class $C^{2}$. Domain $\Omega$ can be either bounded or unbounded. By $\tau$ we denote the distance from a point to the boundary of $\Omega$ measured along the inward normal. In the case of an unbounded domain $\Omega$ we assume that there exists $\tau_{0}>0$ such that variable $\tau$ is well-defined at least for $0<\tau \leqslant \tau_{0}$. In the case of a bounded domain this condition is implied the smoothness of the boundary.

We assume that in the vicinity of each point $P \in \partial \Omega$ we can introduce local coordinates $s=\left(s_{1}, \ldots, s_{n-1}\right)$ with the following properties: point $s=0$ corresponds to point $P$, coordinates $\left(s_{1}, \ldots, s_{n-1}\right)$ are orthogonal at point $P$ and there exists constant $\delta>0$ independent on the choice of point $P$ such that variables $s$ are well-defined at least in $\left\{x: 0 \leqslant s_{i} \leqslant \delta, i=\right.$ $\left.1, \ldots, n-1,0<\tau \leqslant \tau_{0} / 2\right\}$, while the Jacobians of passage from variables $x$ to variables $(s, \tau)$ and back are uniformly bounded by some constant for all points and this constant is independent on $P$. In the case of a bounded domain the latter condition is implied the smoothness of the boundary.

By $\varepsilon$ we denote a small positive parameter, $\eta=\eta(\varepsilon)$ is some bounded positive function.

Suppose that the boundary of domain $\Omega$ consists of two disjoint parts $\partial \Omega:=\overline{\Upsilon \cup \Xi}$. In set $\Upsilon$ we choose a set of bounded disjoint sets $\gamma_{\varepsilon}^{(i)} \subset \Upsilon, i=1, \ldots, N(\varepsilon)$. If set $\Upsilon$ is bounded, $N(\varepsilon)$ is an integer-valued function, which tends to infinity as $\varepsilon \rightarrow 0$. If set $\Upsilon$ is unbounded, we let $N(\varepsilon)=\infty$ for all $\varepsilon$. We shall assume that the boundaries of $(n-1)$-dimensional domains $\gamma_{\varepsilon}^{(i)}$ consist of a finite number of disjoint closed $(n-2)$-dimensional surfaces of class $C^{2}$.

We denote by $B_{r}(M)$ a ball of radius $r$ centered at point $M$ in $\mathbb{R}^{n}$. We assume that there exist points $M_{\varepsilon}^{i} \in \gamma_{\varepsilon}^{(i)}$ and positive numbers $C_{1}, C_{1}, R_{1}$ and $R_{2}$ independent of $\varepsilon$ such that for $i, j=1, \ldots, N(\varepsilon)$

$$
\begin{gathered}
C_{1} \varepsilon \leqslant \min _{i \neq j}\left|M_{\varepsilon}^{i}-M_{\varepsilon}^{j}\right| \leqslant C_{2} \varepsilon \\
B_{R_{2} \varepsilon \eta}\left(M_{\varepsilon}^{i}\right) \cap \Upsilon \subseteq \gamma_{\varepsilon}^{(i)} \subseteq B_{R_{1} \varepsilon \eta}\left(M_{\varepsilon}^{i}\right), \quad B_{R_{1} \varepsilon \eta}\left(M_{\varepsilon}^{i}\right) \cap B_{R_{1} \varepsilon \eta}\left(M_{\varepsilon}^{j}\right)=\emptyset, \quad i \neq j,
\end{gathered}
$$

hold true. By $A_{i j}=A_{i j}(x), A_{j}=A_{j}(x), A_{0}=A_{0}(x)$ we denote functions defined in domain $\Omega$ and satisfying conditions $A_{i j} \in W_{\infty}^{1}(\Omega), A_{j} \in W_{\infty}^{1}(\Omega), A_{0} \in L_{\infty}(\Omega)$. Functions $A_{i j}$ and $A_{0}$ are assumed to be real valued, $A_{j}$ are complex-valued. Moreover, functions $A_{i j}$ satisfy the ellipticity condition

$$
A_{i j}=\bar{A}_{j i}, \quad \sum_{i, j=1}^{n} A_{i j}(x) z_{i} \bar{z}_{j} \geqslant c_{0} \sum_{i=1}^{n}\left|z_{i}\right|^{2}, \quad x \in \Omega, \quad z_{i} \in \mathbb{C}
$$

where $c_{0}$ is a positive constant independent of $x$ and $z_{i}$.

In the present work we consider operator depending on $\varepsilon$, which we denote by $\mathcal{H}_{\varepsilon}$. This is the operator with the differential expression

$$
-\sum_{i, j=1}^{n} \frac{\partial}{\partial x_{i}} A_{i j} \frac{\partial}{\partial x_{j}}+\sum_{j=1}^{n} A_{j} \frac{\partial}{\partial x_{j}}-\frac{\partial}{\partial x_{j}} \bar{A}_{j}+A_{0}
$$

in $\Omega$ subject to Dirichlet condition on $\gamma_{\varepsilon}$ and to Robin condition

$$
\left(\frac{\partial}{\partial \nu}+a\right) u=0 \quad \text { on } \quad \Gamma_{\varepsilon} \cup \Xi, \quad \frac{\partial}{\partial \nu}:=-\sum_{i, j=1}^{n} A_{i j} \widetilde{\nu}_{j} \frac{\partial}{\partial x_{i}}-\sum_{j=1}^{n} \bar{A}_{j} \widetilde{\nu}_{j},
$$


where $\gamma_{\varepsilon}:=\bigcup_{i=1}^{N(\varepsilon)} \gamma_{\varepsilon}^{(i)}, \Gamma_{\varepsilon}:=\Upsilon \backslash \bar{\gamma}_{\varepsilon}, \widetilde{\nu}=\left(\widetilde{\nu}_{1}, \widetilde{\nu}_{2}, \ldots, \widetilde{\nu}_{n}\right)$ is the inward normal to $\partial \Omega, a=a(x)$ is a some real function defined on $\partial \Omega$ and $a \in W_{\infty}^{1}(\partial \Omega)$.

By $\mathfrak{h}_{\varepsilon}$ we denote a closed symmetric sesquilinear lower-semibounded form

$$
\begin{aligned}
\mathfrak{h}_{\varepsilon}(u, v):= & \sum_{i, j=1}^{n}\left(A_{i j} \frac{\partial u}{\partial x_{j}}, \frac{\partial v}{\partial x_{i}}\right)_{L_{2}(\Omega)}+\sum_{j=1}^{n}\left(A_{j} \frac{\partial u}{\partial x_{j}}, v\right)_{L_{2}(\Omega)} \\
& +\sum_{j=1}^{n}\left(u, A_{j} \frac{\partial v}{\partial x_{j}}\right)_{L_{2}(\Omega)}+\left(A_{0} u, v\right)_{L_{2}(\Omega)}+(a u, v)_{L_{2}\left(\Gamma_{\varepsilon} \cup \Xi\right)}
\end{aligned}
$$

in $L_{2}(\Omega)$ on the domain $\stackrel{\circ}{W}_{2}^{1}\left(\Omega, \gamma_{\varepsilon}\right)$. Here $\stackrel{\circ}{W}_{2}^{1}\left(\Omega, \gamma_{\varepsilon}\right)$ is a subspace of functions $W_{2}^{1}(\Omega)$ vanishing on $\gamma_{\varepsilon}$. By $\stackrel{\circ}{W}_{2}^{1}(\Omega, Q)$ we denote the Sobolev space consisting of functions $W_{2}^{1}(\Omega)$ with zero trace on surface $Q$ lying in domain $\Omega \subset \mathbb{R}^{n}$. We define rigorously $\mathcal{H}_{\varepsilon}$ as a self-adjoint operator in $L_{2}(\Omega)$ associated with form $\mathfrak{h}_{\varepsilon}(u, v)$.

It was said above that in the vicinity of each point $P$ we can introduce local orthogonal coordinates $s$. The coordinates corresponding to points $M_{\varepsilon}^{i}$ will be denoted by $s^{i}=\left(s_{1}^{i}, s_{2}^{i}, \ldots, s_{n-1}^{i}\right)$. The images of sets $\gamma_{\varepsilon}^{(i)}$ in variables $s^{i}$ will be denoted by $\omega_{\varepsilon}^{(i)}$.

We introduce the auxiliary problem

$$
\Delta_{\zeta} Y_{\varepsilon}^{i}=0, \quad \zeta \in S^{+}, \quad Y_{\varepsilon}^{i}=1, \quad \zeta \in \omega^{(i)}, \quad \frac{\partial Y_{\varepsilon}^{i}}{\partial \zeta_{n}}=0, \quad \zeta \in\left\{\zeta: \zeta_{n}=0\right\} \backslash \omega^{(i)},
$$

Here $S^{+}=\left\{\zeta: \zeta_{n}>0\right\}$, and $\omega^{(i)}$ is a set obtained by dilating set $\omega_{\varepsilon}^{(i)}$ in $(\varepsilon \eta)^{-1}$ times. In accordance with [13, Lm. 5.4], the solution to this problem exists and is unique, belongs to $W_{2}^{1}\left(S^{+}\right)$, uniformly bounded for all $\zeta \in S^{+}$, has the differentiable asymptotics

$$
Y_{\varepsilon}^{i}(\zeta)=N_{0}^{i}|\zeta|^{-n+2}+O\left(|\zeta|^{-n+1}\right)
$$

as $\zeta \rightarrow \infty$ and satisfies the inequalities

$$
\begin{aligned}
& \left|\frac{\partial Y_{\varepsilon}^{i}}{\partial \zeta_{j}}\right| \leqslant C|\zeta|^{-n+1},\left.\left.\quad\left|Y_{\varepsilon}^{i}-N_{0}^{i}\right| \zeta\right|^{-n+2}|\leqslant C| \zeta\right|^{-n+1} \quad \text { in } \quad\left\{\zeta: \zeta_{n}>0,|\zeta| \geqslant \delta\right\}, \\
& \left|Y_{\varepsilon}^{i}\right| \leqslant C \quad \text { in } \quad\left\{\zeta: \zeta_{n}>0,|\zeta|<\delta\right\} .
\end{aligned}
$$

Here $\delta$ is such that the inclusion $\omega^{(i)} \subset\left\{\zeta: \zeta_{n}=0,\left|\zeta^{\prime}\right|<\delta\right\}$ holds true, while function $Y_{\varepsilon}^{i}$, coefficient $N_{0}^{i}$ and constants $C$ in the latter inequalities depend, generally speaking, on set $\omega^{(i)}$.

We also assume that

$(C 1)$. Coefficients $N_{0}^{i}$ in (2.6) and constants $C$ in (2.7) are uniformly bounded in $i$ and $\varepsilon$.

For each $R_{3}>0$, by $\alpha_{\varepsilon}$ we denote

$$
\alpha_{\varepsilon}(s):= \begin{cases}N_{0}^{i}, & \left|s^{i}\right|<R_{3} \varepsilon, \\ 0, & \text { in other points of domain } \Omega .\end{cases}
$$

In what follows, we shall show in Lemma 3.5 that there exist points $M^{p}$ on boundary $\Upsilon$ and domains $Q_{p}:=\left\{x: 0<s_{j}^{p}<b, j=1, \ldots, n-1, \tau=0\right\}, b>0$, such that the inclusion $\bigcup_{p} Q_{p} \supset \Upsilon$ holds true. Here $s^{p}=\left(s_{1}^{p}, \ldots, s_{n-1}^{p}\right)$ are orthogonal coordinates associated with points $M^{p} \in \Upsilon$.

We impose one more condition. 
(C2). There exists a function $\alpha \in W_{\infty}^{1}(\partial \Omega)$ and function $\kappa=\kappa(\varepsilon), \kappa(\varepsilon) \rightarrow+0, \varepsilon \rightarrow+0$, such that for all sufficiently small $\varepsilon$ the uniform in $p$ estimate

$$
\sum_{q \in \mathbb{Z}^{n-1}} \frac{1}{|q|+1}\left|\int_{Q_{p}}\left(\alpha_{\varepsilon}-\alpha\right) \mathrm{e}^{\frac{2 \pi \mathrm{i}}{b} q \cdot s^{p}} d s\right|^{2} \leqslant \kappa^{2}(\varepsilon),
$$

where $\cdot$ is the scalar product in $\mathbb{R}^{n-1}$. If the intersection of sets $\bar{\Upsilon}$ and $\bar{\Xi}$ is non-empty, then function $\alpha$ vanishes on $\bar{\Upsilon} \cap \bar{\Xi}$.

Throughout the work we assume that

$$
\lim _{\varepsilon \rightarrow 0} \frac{\eta^{n-2}(\varepsilon)}{\varepsilon}=K_{0}
$$

where $K_{0}>0$ is a some constant.

The aim of the work is to study the asymptotic behavior of the resolvent of operator $\mathcal{H}_{\varepsilon}$ as $\varepsilon \rightarrow 0$. We denote $K:=\alpha K_{0} G_{n}$, where $G_{n}=\frac{2 \sqrt{\pi} \Gamma\left(\frac{n+1}{2}\right)}{\Gamma\left(\frac{n}{2}\right)}, \Gamma$ is the Gamma function, while function $\alpha$ is defined in $(\mathrm{C} 2)$. We define $\mathcal{H}_{0}$ as operator in $L_{2}(\Omega)$ with differential expression (2.4) and subject to boundary conditions

$$
\left(\frac{\partial}{\partial \nu}+a\right) u=0 \quad \text { on } \quad \Xi, \quad\left(\frac{\partial}{\partial \nu}+a+K\right) u=0 \quad \text { on } \Upsilon
$$

We introduce rigorously $\mathcal{H}_{0}$ as the self-adjoint operator in $L_{2}(\Omega)$ associated with the closed symmetric sesquilinear lower-semibounded form $\mathfrak{h}_{0}(u, v):=\mathfrak{h}_{\varepsilon}(u, v)+(K u, v)_{L_{2}(\Upsilon)}$ in $L_{2}(\Omega)$ with domain $W_{2}^{1}(\Omega)$. By analogy with [14, Lm. 3.2], [8, Ch. 3, Sect. 7,8] one can prove that the domain of operator $\mathcal{H}_{0}$ reads as

$$
D\left(\mathcal{H}_{0}\right)=\left\{u \in W_{2}^{2}(\Omega) \text { text: conditions(2.11) holdtrue }\right\} .
$$

We denote

$$
\mu:=\mu(\varepsilon)=\frac{\eta^{n-2}}{\varepsilon}-K_{0} .
$$

By $\|\cdot\|_{X \rightarrow Y}$ we denote the norm of operator acting from Banach space $X$ into Banach space $Y$. The first main result is the following theorem.

Theorem 2.1. Let $D$ be a compact set in the complex plane not intersecting with the spectrum of operator $\mathcal{H}_{0}, \lambda \in D$. There exists function $W_{\varepsilon}$ defined in (3.3) such that for all sufficiently small $\varepsilon$ the inequalities

$$
\begin{aligned}
& \left\|\left(\mathcal{H}_{\varepsilon}-\lambda\right)^{-1}-\left(\mathcal{H}_{0}-\lambda\right)^{-1}\right\|_{L_{2}(\Omega) \rightarrow L_{2}(\Omega)} \leqslant C(\lambda, D)\left(\varepsilon^{\frac{1}{2}}+\mu+\kappa(\varepsilon)\right), \\
& \left\|\left(\mathcal{H}_{\varepsilon}-\lambda\right)^{-1}-\left(1-W_{\varepsilon}\right)\left(\mathcal{H}_{0}-\lambda\right)^{-1}\right\|_{L_{2}(\Omega) \rightarrow W_{2}^{1}(\Omega)} \leqslant C(\lambda, D)\left(\varepsilon^{\frac{1}{2}}+\mu+\kappa(\varepsilon)\right)
\end{aligned}
$$

hold true, where constant $C$ is independent of $\varepsilon, \eta$ and $W_{\varepsilon}$ but depends on $\lambda$ and on the choice of compact set $D$.

The second part of the work is devoted to constructing the complete asymptotic expansion for the resolvent of the perturbed operator restricted on the functions in $L_{2}(\Omega)$ with additional smoothness conditions. The asymptotics of the resolvent for an unbounded domain $\Omega$ lying in the upper half-plane. Namely, we assume that there exists $\tau_{0}>0$ such that $\Omega \cap\left\{x: 0<x_{n}<\right.$ $\left.\tau_{0}\right\}=\left\{x: 0<x_{n}<\tau_{0}\right\}$. In addition we assume that the boundary of domain is piece-wise continuous and consists of two disjoint parts: $\partial \Omega=\Upsilon \cup \Xi$, where $\Upsilon:=\left\{x: x_{n}=0\right\}$. We assume also that the coefficients of the perturbed operator and function $a(x)$ are infinitely differentiable as $0 \leqslant x_{n} \leqslant \tau_{0}$, and all these derivatives and these functions are uniformly bounded $0 \leqslant x_{n} \leqslant \tau_{0}$. We shall assume that on boundary $\Upsilon$ functions $A_{i j}(x)$ vanish as $i \neq j$ 
and is equal to $i=j$. Here it is convenient to count the points $M_{\varepsilon}^{i}$ not by index $i$ but multiindex $k \in \mathbb{Z}^{n-1}$ and these points read as $M_{\varepsilon}^{k}=\left(\varepsilon a_{1} k_{1}, \ldots, \varepsilon a_{n-1} k_{n-1}\right)$. We denote by $\gamma$ a set of dimension $(n-1)$ with boundary in class $C^{2}$ lying in $(n-1)$-dimensional parallelepiped $\left\{x \in \mathbb{R}^{n-1}:-\frac{a_{i}}{2}<x_{i}<\frac{a_{i}}{2}, i=1, \ldots, n-1, x_{n}=0\right\}$, where $a_{i}$ are positive constants. We let

$$
\gamma_{\varepsilon}:=\bigcup_{k}\left\{x \in \mathbb{R}^{n-1}:(\varepsilon \eta)^{-1}\left(x-M_{\varepsilon}^{k}\right) \in \gamma, \quad k \in \mathbb{Z}^{n-1}\right\} .
$$

We also note that in this part of the work the additional coefficient in the boundary condition on $\Upsilon$ of the limiting operator is $K:=\frac{N_{0}\left(\mu+K_{0}\right)}{T_{0}}$, see the constructions of the fourth and fifth sections. Here $N_{0}$ is the coefficient at the leading term in the asymptotics at infinity of the solution to an auxiliary problem (4.24). While constant $T_{0}$ is represented by the identity $T_{0}=-\frac{1}{\left|C_{n}\right|} \prod_{i=1}^{n-1} a_{i}$, where $\left|C_{n}\right|$ is the surface area of the unit half-sphere in $\mathbb{R}^{n-1}$.

Let $f$ is an arbitrary function in $L_{2}(\Omega) \cap W_{2}^{m}\left(\left\{x: 0<x_{n}<\tau_{0}\right\}\right)$ for all $m \in \mathbb{N}, u_{\varepsilon}:=$ $\left(\mathcal{H}_{\varepsilon}-\lambda\right)^{-1} f$.

The second main result of the work is as follows.

Theorem 2.2. The asymptotics of function $u_{\varepsilon}$ in norm of $W_{2}^{1}(\Omega)$ is

$$
\begin{aligned}
u_{\varepsilon}(x, \mu, \eta)= & \left(u_{\varepsilon}^{\mathrm{ex}}(x, \mu, \eta)+\chi_{0}\left(x_{n}\right) u_{\varepsilon}^{\mathrm{bl}}\left(\frac{x}{\varepsilon}, x^{\prime}, \mu, \eta\right)\right) \prod_{k \in \mathbb{Z}^{n-1}} \chi_{1}\left(\left|x-M_{\varepsilon}^{k}\right| \varepsilon^{-1} \eta^{-1 / 2}\right) \\
& +\sum_{k \in \mathbb{Z}^{n-1}}\left(1-\chi_{1}\left(\left|x-M_{\varepsilon}^{k}\right| \varepsilon^{-1} \eta^{-1 / 2}\right)\right) u_{\varepsilon}^{\mathrm{in}}\left(x^{\prime}, \mu, \frac{x-M_{\varepsilon}^{k}}{\varepsilon \eta}, \eta\right),
\end{aligned}
$$

where $\chi_{0}\left(x_{n}\right)$ is an infinitely differentiable cut-off function vanishing as $x_{n}>\tau_{0}$ and being equal to one as $x_{n}<\frac{\tau_{0}}{3}, \chi_{1}(t)$ is an infinitely differentiable cut-off function vanishing as $t<1$ and being equal to $t>2$, while the symbols $u_{\varepsilon}^{\mathrm{ex}}, u_{\varepsilon}^{\mathrm{bl}}, u_{\varepsilon}^{\mathrm{in}}$ denote the asymptotic series

$$
\begin{aligned}
& u_{\varepsilon}^{\mathrm{ex}}(x, \mu, \eta)=\sum_{q_{\varepsilon}, q_{\eta}=0}^{\infty} \sum_{q_{l}=0}^{Q_{q_{\varepsilon}, q_{\eta}}} \varepsilon^{q_{\varepsilon}} \eta^{q_{\eta}} \ln ^{\vartheta q_{l}} \eta u_{q_{\varepsilon}, q_{\eta}, q_{l}}(x, \mu), \\
& u_{\varepsilon}^{\mathrm{bl}}\left(x^{\prime}, \mu, \xi, \eta\right)=\mathrm{e}^{\rho\left(x^{\prime}\right) x_{n}} \sum_{q_{\varepsilon}=1}^{\infty} \sum_{q_{\eta}=0}^{\infty} \sum_{q_{l}=0}^{Q_{q_{\varepsilon}, q_{\eta}}-1} \varepsilon^{q_{\varepsilon}} \eta^{q_{\eta}} \ln ^{q_{l}} \eta v_{q_{\varepsilon}, q_{\eta}, q_{l}}\left(x^{\prime}, \mu, \xi\right), \\
& u_{\varepsilon}^{\mathrm{in}}\left(x^{\prime}, \mu, \zeta, \eta\right)=\mathrm{e}^{\rho\left(x^{\prime}\right) x_{n}} \sum_{q_{\varepsilon}, q_{\eta}=0}^{\infty} \sum_{q_{l}=0}^{Q_{q_{\varepsilon}, q_{\eta}}} \varepsilon^{q_{\varepsilon}} \eta^{q_{\eta}} \ln ^{\vartheta q_{l}} \eta w_{q_{\varepsilon}, q_{\eta}, q_{l}}\left(x^{\prime}, \mu, \zeta\right),
\end{aligned}
$$

where $\rho\left(x^{\prime}\right):=\bar{A}_{n}\left(x^{\prime}, 0\right)-a\left(x^{\prime}\right), \vartheta$ is equal to zero as $\left(q_{\varepsilon}, q_{\eta}\right)=(0,0)$ and to one in other cases, $Q_{q_{\varepsilon}, q_{\eta}}:=\min \left(q_{\varepsilon}, q_{\eta}\right)$. The coefficients of series (2.16), 2.17) and (2.18) are determined by Lemmata 4.2 and 4.4. In particular, function $v_{1,0,0}$ is of the form

$$
v_{1,0,0}\left(x^{\prime}, \mu, \xi\right)=-\left.\left(\frac{\partial}{\partial \nu}+a\right) u_{0,0,0}\right|_{x_{n}=0} X(\xi),
$$

where function $X$ is introduced as the solution to the auxiliary problem (4.11). Function $u_{0,0,0}$ is the solution to problem

$$
\begin{gathered}
(\mathcal{L}-\lambda) u_{0,0,0}=f, \quad x \in \Omega \\
\left(\frac{\partial}{\partial \nu}+a+K\right) u_{0,0,0}=\varphi, \quad x \in \Upsilon, \quad\left(\frac{\partial}{\partial \nu}+a\right) u_{0,0,0}=0, \quad x \in \Xi .
\end{gathered}
$$


in $W_{2}^{2}(\Omega)$. Function $w_{0,0,0}$ is of the form $w_{0,0,0}\left(x^{\prime}, \mu, \zeta\right)=u_{0,0,0}\left(x^{\prime}, 0, \mu\right)(1-Y(\zeta))$.

Let us discuss briefly the results of the work. We stress first of all that the result of Theorem 2.1 is true for an arbitrary structure of the frequent alternation of boundary conditions. There are no essential restrictions for the parts of the selected Dirichlet part of boundary. The amount of the parts of these subsets can be finite or infinite, while the result of the theorem is true no matter whether the domain is bounded or not.

We note that in the statement of Theorem 2.1 we have only the norm resolvent convergence of the perturbed operator to the homogenized one in the sense of the norm $\|\cdot\|_{L_{2}(\Omega) \rightarrow L_{2}(\Omega)}$, while the convergence in the sense of the norm $\|\cdot\|_{L_{2}(\Omega) \rightarrow W_{2}^{1}(\Omega)}$ is impossible. Indeed, let $f$ be a function in $L_{2}(\Omega)$. Then for $u_{\varepsilon}:=\left(\mathcal{H}_{\varepsilon}-\lambda\right)^{-1} f$ and $u_{0}:=\left(\mathcal{H}_{0}-\lambda\right)^{-1} f$ we write the corresponding integral identities:

$$
\mathfrak{h}_{\varepsilon}\left(u_{\varepsilon}, u_{\varepsilon}\right)-\lambda\left(u_{\varepsilon}, u_{\varepsilon}\right)_{L_{2}(\Omega)}=\left(f, u_{\varepsilon}\right)_{L_{2}(\Omega)}, \quad \mathfrak{h}_{0}\left(u_{0}, u_{0}\right)-\lambda\left(u_{0}, u_{0}\right)_{L_{2}(\Omega)}=\left(f, u_{0}\right)_{L_{2}(\Omega)} .
$$

We assume that the resolvent of the perturbed operator converges to the resolvent of homogenized one in the norm of operators acting from $L_{2}(\Omega)$ into $W_{2}^{1}(\Omega)$, then $u_{\varepsilon}$ converge to $u_{0}$ in the norm of $W_{2}^{1}(\Omega)$. Therefore, all the terms in the first integral identity converge to similar terms in the second integral identity. But in the left hand side of second integral identity there is one more term $\left(K u_{0}, u_{0}\right)_{L_{2}(\Upsilon)}$ and this is why the original assumption on the convergence of $u_{\varepsilon}$ to $u_{0}$ is wrong. At the same time, the convergence in the norm $\|\cdot\|_{L_{2}(\Omega) \rightarrow L_{2}(\Omega)}$ is sufficient to prove the convergence of the spectrum of the perturbed operator to the spectrum of the homogenized one. Moreover, if we employ a special boundary corrector, we can obtain the convergence in the norm $\|\cdot\|_{L_{2}(\Omega) \rightarrow W_{2}^{1}(\Omega)}$, see (2.14).

Theorem 2.1 states not only the presence of the norm resolvent convergence, but it also gives the estimate for the convergence rate, see (2.13), (2.14). The right hand sides in these inequalities tend to zero due to (2.10), (2.12) and Assumption (C2).

Let us discuss Assumption (C2). In this assumption we introduce function $\alpha$, which, in its turn, determines function $K$. This function in boundary condition (2.11) for the homogenized operator describes the distribution of selected Dirichlet subsets. For a periodic structure of alternating boundary conditions, function $K$ exists and estimate (2.9) holds true for $\kappa(\varepsilon)=C \varepsilon^{\frac{1}{4}}$, where constant $C$ is independent of $\varepsilon$; this will be proved in fifth section. Here the alternation is chosen the same as in Theorem 2.1. In the case of non-periodic structure we can mention the example, when we consider a periodic structure of alternating boundary conditions, but the we change a little the geometry and location of the selected subsets with Dirichlet condition so that the total amount of changed sets is relatively less than the amount of unchanged sets. Then inequality (2.9) still remains true. We also note that assumption (C2) is needed to prove Lemma 3.5, which, in its turn, is employed in the proof of Theorem 2.1. The this lemma is based on sharp estimates. And this gives an opportunity to suppose that the final estimate in this lemma is sharp. This is why term $\kappa(\varepsilon)$ in inequalities (2.13), (2.14) for the resolvent is likely to be also sharp.

The second main result, Theorem 2.2, gives a complete asymptotic expansion for the resolvent. In order to construct such asymptotics, we have to assume additional smoothness for functions $A_{i j}, A_{j}, A_{0}, a$ and $f$ in the vicinity of the part of the boundary with the alternation of boundary conditions. We also assume in addition, that the alternation of boundary conditions is strictly periodic and is imposed on the hyperplane. The coefficients of the series are constructed as the solutions to some sequence of the problems, see the arguments of the forth section.

Let us discuss also an additional coefficient in boundary condition (2.19). This constant $K$ can be calculated by two ways. First, it is determined by the matching condition of asymptotics for function $u_{\varepsilon}$ and also identities (4.26), (4.28). In particular, it will be shown in the end of 
the forth section that the additional coefficient in identity (4.28) is equal to constant $K$. In the second way, coefficient $K$ can be determined by means of Assumption (C2) for the periodic structure of alternation of boundary conditions, see fifth section. Here we also employ the identities for $G_{n}$ and $T_{0}$. We note that the constants determined in both cases will coincide.

\section{Resolvent CONVERGENCE}

In the present section we prove Theorem 2.1 .

Let $f \in L_{2}(\Omega)$ be an arbitrary function, $u_{\varepsilon}:=\left(\mathcal{H}_{\varepsilon}-\lambda\right)^{-1} f, u_{0}:=\left(\mathcal{H}_{0}-\lambda\right)^{-1} f$. We introduce the matrix

$$
A(x):=\left(\begin{array}{cccc}
A_{11}(x) & A_{12}(x) & \ldots & A_{1 n}(x) \\
A_{21}(x) & A_{22}(x) & \ldots & A_{2 n}(x) \\
\vdots & \vdots & \ddots & \vdots \\
A_{n 1}(x) & A_{n 2}(x) & \ldots & A_{n n}(x)
\end{array}\right), \quad A_{\varepsilon}^{i}:=A\left(M_{\varepsilon}^{i}\right),
$$

and for each $i=1, \ldots, N(\varepsilon)$ by $Q_{\varepsilon}^{i}$ we define one matrix by the conditions

$$
\left(Q_{\varepsilon}^{i}\right)^{T}\left(Q_{\varepsilon}^{i}\right)=\left(A_{\varepsilon}^{i}\right)^{-1}, \quad\left(Q_{\varepsilon}^{i}\right)^{T}\left(A_{\varepsilon}^{i}\right)\left(Q_{\varepsilon}^{i}\right)=E,
$$

where $E$ is the unit matrix. Matrix $Q_{\varepsilon}^{i}$ is determined non-uniquely up to the multiplication by an orthogonal matrix. It is easy to check that this orthogonal matrix can be chosen so that $y_{n}^{i}=\tau$, where $y_{n}^{i}$ is $n$-th coordinate of the vector $y^{i}=\left(y_{1}^{i}, \ldots, y_{n-1}^{i}, y_{n}^{i}\right)=Q_{\varepsilon}^{i} z^{i}, z^{i}=\left(s^{i}, \tau\right)$. Hereafter matrix $Q_{\varepsilon}^{i}$ is chosen exactly in this way. By condition (2.3), matrix $A$ is symmetric, lower-semibounded and uniformly bounded for all $x \in \bar{\Omega}$. This is why matrix $Q_{\varepsilon}^{i}$ is lowersemibounded and is uniformly bounded for all $i$ and $\varepsilon$. Therefore, we have the estimate

$$
0<C|z| \leqslant\left|Q_{\varepsilon}^{i} z\right| \leqslant C^{-1}|z|
$$

for all $i=1, \ldots, N(\varepsilon)$, small $\varepsilon$ and $z \in \mathbb{R}^{n}$. Here constant $C$ is independent of $\varepsilon, i$ and $z$.

In the previous section, in the vicinity of each point $M_{\varepsilon}^{i}$ on the boundary of domain $\Omega$ we introduced orthogonal coordinates $s^{i}$. As a result of the passage from variables $x$ to variables $y^{i}$, operator $\nabla$ is recalculated as follows: $\nabla=J_{1}^{i} \nabla_{y^{i}}$, where $J_{1}^{i}$ is some matrix satisfying the inequalities

$$
\left|J_{1}^{i}-E\right| \leqslant C\left|y^{i}\right|, \quad\left|\frac{\partial J_{1}^{i}}{\partial y_{j}^{i}}\right| \leqslant C, \quad j=1, \ldots, n,
$$

where constant $C$ is independent of $y^{i}, i$ and $\varepsilon$. Let $\omega_{\varepsilon}:=\bigcup_{i=1}^{N(\varepsilon)} \omega_{\varepsilon}^{(i)}$, where, we recall, sets $\omega_{\varepsilon}^{(i)}$ we introduced in the previous section as the images of sets $\gamma_{\varepsilon}^{(i)}$.

We denote $B_{\varepsilon}^{i}:=\left\{x:\left|y^{i}\right|<R_{3} \varepsilon\right\} \cap \Omega$. Here $R_{3}$ is a positive constant such that for all $\varepsilon$, $i=1, \ldots, N(\varepsilon)$ the inclusions $\omega_{\varepsilon}^{(i)} \subset B_{\varepsilon}^{i} \subseteq B_{\frac{R_{1 \varepsilon}}{2}}\left(M_{\varepsilon}^{i}\right)$ hold true.

Let $\chi_{2}(t)$ is an infinitely differentiable cut-off function equalling to one as $t<\frac{1}{2}$ and vanishing as $t>1$. Let

$$
\begin{gathered}
Z_{\varepsilon}^{i}(x, \eta):=N_{0}^{i} \eta^{n-2}\left(\varepsilon^{n-2}\left|y^{i}\right|^{-n+2}-R_{3}^{-n+2}\right), \\
W_{\varepsilon}^{i}(x, \eta):=\left(\chi_{2}\left(R_{3}^{-1} \varepsilon^{-1}\left|y^{i}\right|\right) Y_{\varepsilon}^{i}\left(\frac{y^{i}}{\varepsilon \eta}\right)+\left(1-\chi_{2}\left(R_{3}^{-1} \varepsilon^{-1}\left|y^{i}\right|\right)\right) Z_{\varepsilon}^{i}(x, \mu, \eta)\right), \\
W_{\varepsilon}(x, \eta):=\left\{\begin{array}{cl}
W_{\varepsilon}^{i}(x, \eta), & x \in B_{\varepsilon}^{i}, \quad i=1, \ldots, N(\varepsilon), \\
0, & \text { in other points of domain } \bar{\Omega} .
\end{array}\right.
\end{gathered}
$$


By the definition of functions $\chi_{2}$ and $Y_{\varepsilon}^{i}$, function $W_{\varepsilon}$ is continuous in $\bar{\Omega}$, uniformly bounded for all $x \in \bar{\Omega}$ and satisfies the inequality

$$
0 \leqslant W_{\varepsilon} \leqslant 1 .
$$

Hereinafter by $C$ we denote inessential constants independent of $f, u_{0}, u_{\varepsilon}, W_{\varepsilon}, \varepsilon, \eta$ and $x$. In the case of local estimates in the vicinities of sets $\omega_{\varepsilon}^{(i)}$ constants $C$ are independent of the choice of a particular set $\omega_{\varepsilon}^{(i)}$. In accordance with [28, Ch. 5, Sect. 5.3, Ineq. (5.3)], we have

$$
\left\|u_{0}\right\|_{L_{2}(\Omega)} \leqslant C\|f\|_{L_{2}(\Omega)} .
$$

Lemma 3.1. For each function $u \in \stackrel{\circ}{W}_{2}^{1}\left(\Omega, \gamma_{\varepsilon}\right)$ the estimate

$$
\|u\|_{W_{2}^{1}(\Omega)}^{2} \leqslant C\left(\mathfrak{h}_{\varepsilon}(u, u)+\|u\|_{L_{2}(\Omega)}^{2}\right)
$$

holds true.

This lemma can be proved in the same way as [13, Lm. 3.1].

Lemma 3.2. For each function $u \in D\left(\mathfrak{h}_{0}\right)$, the estimate

$$
\|u\|_{W_{2}^{2}(\Omega)} \leqslant C\left(\left\|\mathcal{H}_{0} u\right\|_{L_{2}(\Omega)}+\|u\|_{L_{2}(\Omega)}\right)
$$

holds true.

The proof of this lemma is similar to [13, Lm. 3.2]. In the proof of this lemma we also employ the results of [29, Ch. 3, Sect. 7,8].

In accordance with the definition, function $u_{\varepsilon}$ satisfies the integral identity

$$
\mathfrak{h}_{\varepsilon}\left(u_{\varepsilon}, u_{\varepsilon}\right)+\lambda\left(u_{\varepsilon}, u_{\varepsilon}\right)_{L_{2}(\Omega)}=\left(f, u_{\varepsilon}\right)_{L_{2}(\Omega)} \text {. }
$$

In addition we assume that $\lambda$ has a non-zero imaginary part. In accordance with [28, Ch. 5 , Sect. 5.3, Ineq. (5.3)], for such $\lambda$ the esimate

$$
\left\|u_{\varepsilon}\right\|_{L_{2}(\Omega)} \leqslant C\|f\|_{L_{2}(\Omega)}
$$

holds true. It follows from Lemma 3.1 that

$$
C_{1}\left\|u_{\varepsilon}\right\|_{W_{2}^{1}(\Omega)}^{2} \leqslant \mathfrak{h}_{\varepsilon}\left(u_{\varepsilon}, u_{\varepsilon}\right) \leqslant C\|f\|_{L_{2}(\Omega)}^{2} .
$$

It implies the inequality

$$
\left\|u_{\varepsilon}\right\|_{W_{2}^{1}(\Omega)} \leqslant C\|f\|_{L_{2}(\Omega)} .
$$

By analogy with [14, Lm. 3.3] one can prove

Lemma 3.3. For each function $v \in W_{2}^{1}(\Omega)$, the inequalities

$$
\sum_{i=1}^{N(\varepsilon)}\|v\|_{L_{2}\left(\partial B_{\varepsilon}^{i}\right)}^{2} \leqslant C\|v\|_{W_{2}^{1}(\Omega)}^{2}, \quad \sum_{i=1}^{N(\varepsilon)}\|v\|_{L_{2}\left(B_{\varepsilon}^{i}\right)}^{2} \leqslant C \varepsilon\|v\|_{W_{2}^{1}(\Omega)}^{2},
$$

hold true, where constant $C$ is independent of $\varepsilon$ and $v$.

Lemma 3.4. Function $u_{0} W_{\varepsilon}$ belongs to $W_{2}^{1}(\Omega)$ and this functions satisfies the estimates

$$
\begin{aligned}
& \left\|u_{0} W_{\varepsilon}\right\|_{L_{2}(\Omega)} \leqslant C \varepsilon^{\frac{1}{2}}\|f\|_{L_{2}(\Omega)}, \\
& \left\|W_{\varepsilon} \nabla u_{0}\right\|_{L_{2}(\Omega)} \leqslant C \varepsilon^{\frac{1}{2}}\|f\|_{L_{2}(\Omega)}, \\
& \left\|u_{0} \nabla W_{\varepsilon}\right\|_{L_{2}(\Omega)} \leqslant C\|f\|_{L_{2}(\Omega)},
\end{aligned}
$$

where constant $C$ is independent of $\varepsilon, u_{0}, f$ and $W_{\varepsilon}$. 
Proof. It is sufficient to prove the lemma for real-valued $u_{0} \in C^{\infty}(\bar{\Omega})$ with a compact support, since the linear combinations of such functions are dense in space $W_{2}^{2}(\Omega)$. By the properties of function $Y_{\varepsilon}^{i}$, functions $W_{\varepsilon} u_{0}, W_{\varepsilon} \nabla u_{0}$ belong to $L_{2}(\Omega)$ and it follows from the definition of function $W_{\varepsilon}$, inequality (3.4), Lemmata 3.2, 3.3 that

$$
\begin{aligned}
& \left\|W_{\varepsilon} \nabla u_{0}\right\|_{L_{2}(\Omega)}^{2} \leqslant C \sum_{i=1}^{N(\varepsilon)} \int_{B_{\varepsilon}^{i}}\left|W_{\varepsilon}\right|^{2}\left|\nabla u_{0}\right|^{2} d x \leqslant C \sum_{i=1}^{N(\varepsilon)}\left\|\nabla u_{0}\right\|_{L_{2}\left(B_{\varepsilon}^{i}\right)}^{2} \leqslant C \varepsilon\|f\|_{L_{2}(\Omega)}^{2}, \\
& \left\|W_{\varepsilon} u_{0}\right\|_{L_{2}(\Omega)}^{2} \leqslant C \sum_{i=1}^{N(\varepsilon)} \int_{B_{\varepsilon}^{i}}\left|W_{\varepsilon}\right|^{2}\left|u_{0}\right|^{2} d x \leqslant C \sum_{i=1}^{N(\varepsilon)}\left\|u_{0}\right\|_{L_{2}\left(B_{\varepsilon}^{i}\right)}^{2} \leqslant C \varepsilon\|f\|_{L_{2}(\Omega)}^{2}, \\
& \left\|u_{0} \nabla W_{\varepsilon}\right\|_{L_{2}(\Omega)}^{2} \leqslant C \sum_{i=1}^{N(\varepsilon)} \int_{B_{\varepsilon}^{i}} u_{0}^{2}\left|\nabla W_{\varepsilon}\right|^{2} d x .
\end{aligned}
$$

The first two inequalities in the statement of the lemma are proved. It remains to check that $u_{0} \nabla W_{\varepsilon} \in L_{2}(\Omega)$ and to prove inequality (3.10).

We pass to variables $y^{i}$ in integral $\int u_{0}^{2}\left|\nabla W_{\varepsilon}\right|^{2} d x$. The passage to variables $y^{i}$ make one-toone correspondence between sets $B_{\varepsilon}^{i} \stackrel{B_{\varepsilon}^{i}}{\widetilde{B}_{\varepsilon}^{i}}:=\left\{y^{i}:\left|y^{i}\right|<R_{3} \varepsilon, y_{n}^{i}>0\right\}$. In accordance with the definition of function $W_{\varepsilon}$, we integrate by parts as follows:

$$
\begin{aligned}
\int_{\widetilde{B}_{\varepsilon}^{i}} u_{0}^{2}\left|\nabla_{y^{i}} W_{\varepsilon}\right|^{2} d y^{i} & =\int_{\partial \widetilde{B}_{\varepsilon}^{i}} W_{\varepsilon} u_{0}^{2} \frac{\partial W_{\varepsilon}}{\partial \nu} d S-\frac{1}{2} \int_{\widetilde{B}_{\varepsilon}^{i}} \nabla_{y^{i}} u_{0}^{2} \cdot \nabla_{y^{i}} W_{\varepsilon}^{2} d y^{i}+\int_{\widetilde{B}_{\varepsilon}^{i}} W_{\varepsilon} u_{0}^{2} \Delta_{y^{i}} W_{\varepsilon} d y^{i} \\
& =\int_{\partial \widetilde{B}_{\varepsilon}^{i}} W_{\varepsilon} u_{0}^{2} \frac{\partial W_{\varepsilon}}{\partial \nu} d S-\frac{1}{2} \int_{\partial \widetilde{B}_{\varepsilon}^{i}} W_{\varepsilon}^{2} \frac{\partial u_{0}^{2}}{\partial \nu} d S+\frac{1}{2} \int_{\widetilde{B}_{\varepsilon}^{i}} W_{\varepsilon}^{2} \Delta_{y^{i}} u_{0}^{2} d y^{i} \\
& +\int_{\widetilde{B}_{\varepsilon}^{i}} W_{\varepsilon} u_{0}^{2} \Delta_{y^{i}} W_{\varepsilon} d y^{i} .
\end{aligned}
$$

Then by the definition of function $W_{\varepsilon}$, the belongings $u_{0}, \frac{\partial u_{0}}{\partial \nu} \in L_{2}\left(\partial B_{\varepsilon}^{i}\right), u_{0} \in W_{2}^{2}(\Omega)$, inequalities (3.4), (3.8), (3.9), Lemmata 3.2, 3.3, inequalities in (3.2), the boundedness of the Jacobians and (3.11) we obtain

$$
\begin{aligned}
\left\|u_{0} \nabla W_{\varepsilon}\right\|_{W_{2}^{1}(\Omega)}^{2} \leqslant & C \int_{\widetilde{B}_{\varepsilon}^{i}} u_{0}^{2}\left|\nabla_{y^{i}} W_{\varepsilon}\right|^{2} d y^{i} \leqslant C\left(\left(\sum_{i=1}^{N(\varepsilon)}\left\|u_{0}\right\|_{L_{2}\left(\partial B_{\varepsilon}^{i}\right)}^{2}\right)^{\frac{1}{2}}\left(\sum_{i=1}^{N(\varepsilon)}\left\|\frac{\partial u_{0}}{\partial \nu}\right\|_{L_{2}\left(\partial B_{\varepsilon}^{i}\right)}^{2}\right)^{\frac{1}{2}}\right. \\
& +\sum_{i=1}^{N(\varepsilon)}\left\|\nabla u_{0}\right\|_{L_{2}\left(B_{\varepsilon}^{i}\right)}^{2}+\left(\sum_{i=1}^{N(\varepsilon)}\left\|u_{0}\right\|_{L_{2}\left(B_{\varepsilon}^{i}\right)}^{2}\right)^{\frac{1}{2}}\left(\sum_{i=1}^{N(\varepsilon)}\left\|\Delta u_{0}\right\|_{L_{2}\left(B_{\varepsilon}^{i}\right)}^{2}\right)^{\frac{1}{2}} \\
& \left.+\sum_{i=1}^{N(\varepsilon)}\left(\left\|\left(Y_{\varepsilon}^{i}-Z_{\varepsilon}^{i}\right) u_{0} \Delta_{y^{i}} \chi_{2}\right\|_{L_{2}\left(\widetilde{B}_{\varepsilon}^{i}\right)}^{2}+\left\|u_{0} \nabla_{y^{i}}\left(Y_{\varepsilon}^{i}-Z_{\varepsilon}^{i}\right) \nabla_{y^{i}} \chi_{2}\right\|_{L_{2}\left(\widetilde{B}_{\varepsilon}^{i}\right)}^{2}\right)\right) \\
\leqslant & C\left\|u_{0}\right\|_{W_{2}^{2}(\Omega)}^{2} \leqslant C\|f\|_{L_{2}(\Omega)}^{2} .
\end{aligned}
$$

It follows that $u_{0} W_{\varepsilon} \in W_{2}^{1}(\Omega)$ and estimate (3.10) holds true. The proof is complete. 
We denote $\varphi_{\varepsilon}:=u_{\varepsilon}-\left(1-W_{\varepsilon}\right) u_{0}$. By the last lemma and the definition of function $W_{\varepsilon}$ we obtain $\varphi_{\varepsilon} \in \stackrel{\circ}{W}_{2}^{1}\left(\Omega, \omega_{\varepsilon}\right)$. For the resolvents of operators $\mathcal{H}_{\varepsilon}$ and $\mathcal{H}_{0}$ we write the corresponding integral identities choosing $\varphi_{\varepsilon}$ as the test-function

$$
\mathfrak{h}_{\varepsilon}\left(u_{\varepsilon}, \varphi_{\varepsilon}\right)-\lambda\left(u_{\varepsilon}, \varphi_{\varepsilon}\right)_{L_{2}(\Omega)}=\left(f, \varphi_{\varepsilon}\right)_{L_{2}(\Omega)}, \quad \mathfrak{h}_{0}\left(u_{0}, \varphi_{\varepsilon}\right)-\lambda\left(u_{0}, \varphi_{\varepsilon}\right)_{L_{2}(\Omega)}=\left(f, \varphi_{\varepsilon}\right)_{L_{2}(\Omega)} .
$$

Let us calculate the difference of these identities:

$$
\begin{aligned}
0 & =\mathfrak{h}_{\varepsilon}\left(u_{\varepsilon}-u_{0}, \varphi_{\varepsilon}\right)-\lambda\left(u_{\varepsilon}-u_{0}, \varphi_{\varepsilon}\right)_{L_{2}(\Omega)}+\left(K u_{0}, \varphi_{\varepsilon}\right)_{L_{2}(\Upsilon)} \\
& =\mathfrak{h}_{\varepsilon}\left(\varphi_{\varepsilon}, \varphi_{\varepsilon}\right)-\lambda\left\|\varphi_{\varepsilon}\right\|_{L_{2}(\Omega)}^{2}-\mathfrak{h}_{\varepsilon}\left(W_{\varepsilon} u_{0}, \varphi_{\varepsilon}\right)+\lambda\left(u_{0}, \varphi_{\varepsilon} W_{\varepsilon}\right)_{L_{2}(\Omega)}+\left(K u_{0}, \varphi_{\varepsilon}\right)_{L_{2}(\Upsilon)} .
\end{aligned}
$$

It implies the integral identity for function $\varphi_{\varepsilon}$ :

$$
\mathfrak{h}_{\varepsilon}\left(\varphi_{\varepsilon}, \varphi_{\varepsilon}\right)-\lambda\left\|\varphi_{\varepsilon}\right\|_{L_{2}(\Omega)}^{2}=g_{\varepsilon}\left(W_{\varepsilon} u_{0}, \varphi_{\varepsilon}\right)+\left(K u_{0}, \varphi_{\varepsilon}\right)_{L_{2}(\Upsilon)},
$$

where we denote

$$
\begin{aligned}
g_{\varepsilon}\left(W_{\varepsilon} u_{0}, \varphi_{\varepsilon}\right):=K_{1}^{\varepsilon}+K_{2}^{\varepsilon} \\
K_{1}^{\varepsilon}:=\sum_{i=1}^{N(\varepsilon)}\left(A_{\varepsilon}^{i} u_{0} \nabla W_{\varepsilon}, \nabla \varphi_{\varepsilon}\right)_{L_{2}\left(B_{\varepsilon}^{i}\right)}, \\
K_{2}^{\varepsilon}:=\sum_{i=1}^{N(\varepsilon)}\left[\left(A-A_{\varepsilon}^{i}\right) u_{0} \nabla W_{\varepsilon}, \nabla \varphi_{\varepsilon}\right)_{L_{2}\left(B_{\varepsilon}^{i}\right)}-\sum_{j=1}^{n}\left(A_{j} u_{0} \frac{\partial W_{\varepsilon}}{\partial x_{j}}, \varphi_{\varepsilon}\right)_{L_{2}\left(B_{\varepsilon}^{i}\right)} \\
\left.+\left(A W_{\varepsilon} \nabla u_{0}, \nabla \varphi_{\varepsilon}\right)_{L_{2}\left(B_{\varepsilon}^{i}\right)}+\sum_{j=1}^{n}\left(A_{j} W_{\varepsilon} \frac{\partial u_{0}}{\partial x_{j}}, \varphi_{\varepsilon}\right)_{L_{2}\left(B_{\varepsilon}^{i}\right)}^{n}\left(W_{\varepsilon} u_{0}, A_{j} \frac{\partial \varphi_{\varepsilon}}{\partial x_{j}}\right)_{L_{2}\left(B_{\varepsilon}^{i}\right)}\right] .
\end{aligned}
$$

Our next step is to estimate the right hand side in (3.12) and to obtain the estimate for $\varphi_{\varepsilon}$, and then to estimate the norm of function $u_{\varepsilon}-u_{0}$.

We first estimate $K_{2}^{\varepsilon}$. By the definition of function $W_{\varepsilon}$, Lemmata 3.2, 3.3, 3.4 and inequality

$$
\left|A_{i j}(x)-A_{i j}\left(M_{\varepsilon}^{i}\right)\right| \leqslant C \varepsilon \quad \text { in } \quad B_{\varepsilon}^{i},
$$

we get

$$
\begin{aligned}
& \left|K_{2}^{\varepsilon}\right| \leqslant C\left[\varepsilon\left(\sum_{i=1}^{N(\varepsilon)}\left\|u_{0} \nabla W_{\varepsilon}\right\|_{L_{2}\left(B_{\varepsilon}^{i}\right)}^{2}\right)^{\frac{1}{2}}\left(\sum_{i=1}^{N(\varepsilon)}\left\|\varphi_{\varepsilon}\right\|_{W_{2}^{1}\left(B_{\varepsilon}^{i}\right)}^{2}\right)^{\frac{1}{2}}\right. \\
& +\left(\sum_{i=1}^{N(\varepsilon)}\left\|u_{0} \nabla W_{\varepsilon}\right\|_{L_{2}\left(B_{\varepsilon}^{i}\right)}^{2}\right)^{\frac{1}{2}}\left(\sum_{i=1}^{N(\varepsilon)}\left\|\varphi_{\varepsilon}\right\|_{L_{2}\left(B_{\varepsilon}^{i}\right)}^{2}\right)^{\frac{1}{2}}+\left(\sum_{i=1}^{N(\varepsilon)}\left\|W_{\varepsilon} \nabla u_{0}\right\|_{L_{2}\left(B_{\varepsilon}^{i}\right)}^{2}\right)^{\frac{1}{2}}\left(\sum_{i=1}^{N(\varepsilon)}\left\|\varphi_{\varepsilon}\right\|_{W_{2}^{1}\left(B_{\varepsilon}^{i}\right)}^{2}\right)^{\frac{1}{2}} \\
& \left.+\left(\sum_{i=1}^{N(\varepsilon)}\left\|W_{\varepsilon} u_{0}\right\|_{L_{2}\left(B_{\varepsilon}^{i}\right)}^{2}\right)^{\frac{1}{2}}\left(\sum_{i=1}^{N(\varepsilon)}\left\|\varphi_{\varepsilon}\right\|_{W_{2}^{1}\left(B_{\varepsilon}^{i}\right)}^{2}\right)^{\frac{1}{2}}\right] \leqslant C \varepsilon^{\frac{1}{2}}\|f\|_{L_{2}(\Omega)}\left\|\varphi_{\varepsilon}\right\|_{W_{2}^{1}(\Omega)} .
\end{aligned}
$$

We denote $\Gamma_{i}:=\partial B_{\varepsilon}^{i} \cap \partial \Omega, S_{i}:=\partial B_{\varepsilon}^{i} \backslash \partial \Omega$. Employing the definition of function $W_{\varepsilon}$, we integrate by parts as follows:

$$
K_{1}^{\varepsilon}=K_{1,1}^{\varepsilon}+K_{1,2}^{\varepsilon}+K_{1,3}^{\varepsilon},
$$




$$
\begin{aligned}
& K_{1,1}^{\varepsilon}:=\sum_{i=1}^{N(\varepsilon)}\left(\nu \cdot A_{\varepsilon}^{i} \nabla W_{\varepsilon}, u_{0} \varphi_{\varepsilon}\right)_{L_{2}\left(\partial B_{\varepsilon}^{i}\right)}, \quad K_{1,2}^{\varepsilon}:=-\sum_{i=1}^{N(\varepsilon)}\left(\operatorname{div} A_{\varepsilon}^{i} \nabla W_{\varepsilon}, \varphi_{\varepsilon} u_{0}\right)_{L_{2}\left(B_{\varepsilon}^{i}\right)}, \\
& K_{1,3}^{\varepsilon}:=-\sum_{i=1}^{N(\varepsilon)}\left(A_{\varepsilon}^{i} \nabla u_{0}, \varphi_{\varepsilon} \nabla W_{\varepsilon}\right)_{L_{2}\left(B_{\varepsilon}^{i}\right)}=K_{2,1}^{\varepsilon}+K_{2,2}^{\varepsilon}, \\
& K_{2,1}^{\varepsilon}:=\sum_{i=1}^{N(\varepsilon)}\left(\left(A_{\varepsilon}^{i} \nabla u_{0}, W_{\varepsilon} \nabla \varphi_{\varepsilon}\right)_{L_{2}\left(B_{\varepsilon}^{i}\right)}+\left(\operatorname{div} A_{\varepsilon}^{i} \nabla u_{0}, \varphi_{\varepsilon} W_{\varepsilon}\right)_{L_{2}\left(B_{\varepsilon}^{i}\right)}\right), \\
& K_{2,2}^{\varepsilon}:=-\sum_{i=1}^{N(\varepsilon)}\left(\nu \cdot A_{\varepsilon}^{i} \nabla u_{0}, \varphi_{\varepsilon} W_{\varepsilon}\right)_{L_{2}\left(\partial B_{\varepsilon}^{i}\right)}=-\sum_{i=1}^{N(\varepsilon)}\left(\nu \cdot A_{\varepsilon}^{i} \nabla u_{0}, \varphi_{\varepsilon} W_{\varepsilon},\right)_{L_{2}\left(\Gamma_{i}\right)},
\end{aligned}
$$

where $\nu$ is the outward normal to $\partial B_{\varepsilon}^{i}$.

Let us estimate $K_{2,1}^{\varepsilon}$. In accordance wit the definition of function $W_{\varepsilon}$, Lemmata 3.2 , 3.3 and 3.4 and inequality (3.4) we get:

$$
\begin{aligned}
\left|K_{2,1}^{\varepsilon}\right| \leqslant & \left(\left(\sum_{i=1}^{N(\varepsilon)}\left\|W_{\varepsilon} \nabla u_{0}\right\|_{L_{2}\left(B_{\varepsilon}^{i}\right)}^{2}\right)^{\frac{1}{2}}\left(\sum_{i=1}^{N(\varepsilon)}\left\|\nabla \varphi_{\varepsilon}\right\|_{L_{2}\left(B_{\varepsilon}^{i}\right)}^{2}\right)^{\frac{1}{2}}\right. \\
& \left.+\left(\sum_{i=1}^{N(\varepsilon)}\left\|u_{0}\right\|_{W_{2}^{2}\left(B_{\varepsilon}^{i}\right)}^{2}\right)^{\frac{1}{2}}\left(\sum_{i=1}^{N(\varepsilon)}\left\|\varphi_{\varepsilon}\right\|_{L_{2}\left(B_{\varepsilon}^{i}\right)}^{2}\right)^{\frac{1}{2}}\right) \leqslant C \varepsilon^{\frac{1}{2}}\|f\|_{L_{2}(\Omega)}\left\|\varphi_{\varepsilon}\right\|_{W_{2}^{1}(\Omega)} .
\end{aligned}
$$

We rewrite $K_{1,1}^{\varepsilon}$ and $K_{1,2}^{\varepsilon}$ in local variables $y^{i}$. Since $\nabla=J_{1}^{i} \nabla_{y^{i}}$, in accordance with the definition of function $W_{\varepsilon}$, the inequalities in (3.2), Lemmata 3.2, 3.3 and 3.4, inequality (3.4) and the boundedness of Jacobians, we arrive at the estimate for $K_{1,2}^{\varepsilon}$ :

$$
\begin{aligned}
\left|K_{1,2}^{\varepsilon}\right| \leqslant & C\left(\left(\sum_{i=1}^{N(\varepsilon)}\left\|\left(Y_{\varepsilon}^{i}-Z_{\varepsilon}^{i}\right) u_{0} \Delta_{y^{i}} \chi_{2}\right\|_{L_{2}\left(\widetilde{B}_{\varepsilon}^{i}\right)}^{2}\right)^{\frac{1}{2}}\left(\sum_{i=1}^{N(\varepsilon)}\left\|\varphi_{\varepsilon}\right\|_{L_{2}\left(\widetilde{B}_{\varepsilon}^{i}\right)}^{2}\right)^{\frac{1}{2}}\right. \\
& \left.+\left(\sum_{i=1}^{N(\varepsilon)}\left\|u_{0} \nabla_{y^{i}}\left(Y_{\varepsilon}^{i}-Z_{\varepsilon}^{i}\right) \nabla_{y^{i}} \chi_{2}\right\|_{L_{2}\left(\widetilde{B}_{\varepsilon}^{i}\right)}^{2}\right)^{\frac{1}{2}}\left(\sum_{i=1}^{N(\varepsilon)}\left\|\varphi_{\varepsilon}\right\|_{L_{2}\left(\widetilde{B}_{\varepsilon}^{i}\right)}^{2}\right)^{\frac{1}{2}}\right) \\
\leqslant & C \varepsilon\|f\|_{L_{2}(\Omega)}\left(\sum_{i=1}^{N(\varepsilon)}\left\|\varphi_{\varepsilon}\right\|_{L_{2}\left(B_{\varepsilon}^{i}\right)}^{2}\right)^{\frac{1}{2}} \leqslant C \varepsilon^{\frac{3}{2}}\|f\|_{L_{2}(\Omega)}\left\|\varphi_{\varepsilon}\right\|_{W_{2}^{1}(\Omega)} .
\end{aligned}
$$

Let us estimate $K_{2,2}^{\varepsilon}$. By analogy with the proof of Lemma 3.4 in [14], using the definition of function $W_{\varepsilon}$, Lemmata 3.2, 3.3, 3.4 and inequality (3.4), we obtain

$$
\left|K_{2,2}^{\varepsilon}\right| \leqslant C \varepsilon\|f\|_{L_{2}(\Omega)}\left\|\varphi_{\varepsilon}\right\|_{W_{2}^{1}(\Omega)} .
$$

Let $\xi$ be Cartesian coordinates in $\mathbb{R}^{n}, \Sigma:=\left\{\xi:|\xi|<R_{3}, \xi_{n}>0\right\}, S^{1}:=\partial \Sigma \backslash\left\{\xi: \xi_{n}=0\right\}$, $\Gamma^{1}:=\partial \Sigma \cap\left\{\xi: \xi_{n}=0\right\}$. We introduce the problem

$$
\Delta_{\xi} V=0, \quad \xi \in \Sigma, \quad \frac{\partial V}{\partial \nu}=1, \quad \xi \in S^{1}, \quad \frac{\partial V}{\partial \nu}=G_{n}, \quad \xi \in \Gamma^{1},
$$

where $\nu$ is the outward normal for $\partial \Sigma$ and constant $G_{n}$ was introduced in the previous section. The solution to this problem exists and belongs to $W_{2}^{1}(\Sigma)$. Function $V$ and its first derivatives are uniformly bounded for all $\xi \in \bar{\Sigma}$. In the vicinity of each point $M_{\varepsilon}^{i}$ we introduce rescaled 
coordinates $\xi^{i}$ by the rule $\xi^{i}=y^{i} \varepsilon^{-1}$. We denote $\Sigma_{\varepsilon}^{i}:=\left\{y^{i}: \xi^{i} \in \Sigma\right\}, V_{\varepsilon}^{i}\left(y^{i}\right):=V\left(\xi^{i}\right)$, $S_{\varepsilon}^{i}:=\partial \Sigma_{\varepsilon}^{i} \backslash\left\{y^{i}: \xi_{n}^{i}=0\right\}, \Gamma_{\varepsilon}^{i}:=\partial \Sigma_{\varepsilon}^{i} \cap\left\{y^{i}: \xi_{n}^{i}=0\right\}$.

We integrate by parts as follows:

$$
\begin{aligned}
0= & -N_{0}^{i} K_{0} \varepsilon \int_{\widetilde{B}_{\varepsilon}^{i}} u_{0} \varphi_{\varepsilon} \Delta_{y^{i}} V_{\varepsilon}^{i} d y^{i}=-N_{0}^{i} K_{0}\left(u_{0}, \varphi_{\varepsilon}\right)_{L_{2}\left(S_{\varepsilon}^{i}\right)} \\
& -N_{0}^{i} G_{n} K_{0}\left(u_{0}, \varphi_{\varepsilon}\right)_{L_{2}\left(\Gamma_{\varepsilon}^{i}\right)}+N_{0}^{i} K_{0} \varepsilon \int_{\widetilde{B}_{\varepsilon}^{i}} \nabla_{y^{i}} V_{\varepsilon}^{i} \nabla_{y^{i}} u_{0} \varphi_{\varepsilon} d y^{i} .
\end{aligned}
$$

We denote

$$
\begin{aligned}
& K_{1,4}^{\varepsilon}:=K_{1,1}^{\varepsilon}-\sum_{i=1}^{N(\varepsilon)} N_{0}^{i} K_{0}\left(u_{0}, \varphi_{\varepsilon}\right)_{L_{2}\left(S_{\varepsilon}^{i}\right)}, \\
& K_{1,5}^{\varepsilon}:=\sum_{i=1}^{N(\varepsilon)} N_{0}^{i} K_{0} \varepsilon\left(\nabla_{y^{i}} V_{\varepsilon}^{i}, \nabla_{y^{i}} u_{0} \varphi_{\varepsilon}\right)_{L_{2}\left(\widetilde{B}_{\varepsilon}^{i}\right)}, \\
& K_{3}^{\varepsilon}:=\left(K u_{0}, \varphi_{\varepsilon}\right)_{L_{2}(\Upsilon)}-\sum_{i=1}^{N(\varepsilon)} N_{0}^{i} G_{n} K_{0}\left(u_{0}, \varphi_{\varepsilon}\right)_{L_{2}\left(\Gamma_{\varepsilon}^{i}\right)} .
\end{aligned}
$$

We write the estimate for $K_{1,4}^{\varepsilon}$ and $K_{1,5}^{\varepsilon}$. Passing to variables $x$ in integrals $K_{1,4}^{\varepsilon}$ and $K_{1,5}^{\varepsilon}$, in accordance with the definition of functions $W_{\varepsilon}, V_{\varepsilon}^{i}$, inequalities in (3.2), Lemmata 3.2, 3.3 and 3.4, inequality (3.4) and the boundedness of Jacobians we obtain

$$
\begin{aligned}
\left|K_{1,4}^{\varepsilon}\right| \leqslant & C\left(\frac{\eta^{n-2}}{\varepsilon}-K_{0}\right)\left(\sum_{i=1}^{N(\varepsilon)}\left\|u_{0}\right\|_{L_{2}\left(\partial B_{\varepsilon}^{i}\right)}^{2}\right)^{\frac{1}{2}}\left(\sum_{i=1}^{N(\varepsilon)}\left\|\varphi_{\varepsilon}\right\|_{L_{2}\left(\partial B_{\varepsilon}^{i}\right)}^{2}\right)^{\frac{1}{2}} \leqslant C \mu\|f\|_{L_{2}(\Omega)}\left\|\varphi_{\varepsilon}\right\|_{W_{2}^{1}(\Omega)}, \\
\left|K_{1,5}^{\varepsilon}\right| \leqslant & \left(\left(\sum_{i=1}^{N(\varepsilon)}\left\|\nabla u_{0}\right\|_{L_{2}\left(B_{\varepsilon}^{i}\right)}^{2}\right)^{\frac{1}{2}}\left(\sum_{i=1}^{N(\varepsilon)}\left\|\varphi_{\varepsilon}\right\|_{L_{2}\left(B_{\varepsilon}^{i}\right)}^{2}\right)^{\frac{1}{2}}\right. \\
& \left.+\left(\sum_{i=1}^{N(\varepsilon)}\left\|u_{0}\right\|_{L_{2}\left(B_{\varepsilon}^{i}\right)}^{2}\right)^{\frac{1}{2}}\left(\sum_{i=1}^{N(\varepsilon)}\left\|\nabla \varphi_{\varepsilon}\right\|_{L_{2}\left(B_{\varepsilon}^{i}\right)}^{2}\right)^{\frac{1}{2}}\right) \\
\leqslant & C\left(\varepsilon+\varepsilon^{\frac{1}{2}}\right)\|f\|_{L_{2}(\Omega)}\left\|\varphi_{\varepsilon}\right\|_{W_{2}^{1}(\Omega)} \leqslant C \varepsilon^{\frac{1}{2}}\|f\|_{L_{2}(\Omega)}\left\|\varphi_{\varepsilon}\right\|_{W_{2}^{1}(\Omega)} .
\end{aligned}
$$

Then by the obtained estimates for $K_{1,1}^{\varepsilon}, K_{1,2}^{\varepsilon}, K_{1,3}^{\varepsilon}, K_{1,4}^{\varepsilon}, K_{1,5}^{\varepsilon}, K_{2,1}^{\varepsilon}$ and $K_{2,2}^{\varepsilon}$ we get the estimate for $K_{1}^{\varepsilon}$ :

$$
\left|K_{1}^{\varepsilon}\right| \leqslant C\left(\varepsilon^{\frac{1}{2}}+\mu\right)\|f\|_{L_{2}(\Omega)}\left\|\varphi_{\varepsilon}\right\|_{W_{2}^{1}(\Omega)} .
$$

Let us estimate $K_{3}^{\varepsilon}$.

Lemma 3.5. The inequality

$$
\left|K_{3}^{\varepsilon}\right| \leqslant C \kappa(\varepsilon)\|f\|_{L_{2}(\Omega)}\left\|\varphi_{\varepsilon}\right\|_{W_{2}^{1}(\Omega)}
$$

holds true, where constant $C$ is independent of $\varepsilon, f$ and $\varphi_{\varepsilon}$.

Proof. We choose arbitrary point $M^{1}$ on boundary $\Upsilon$. In the vicinity of this point we introduce orthogonal coordinates $s^{1}=\left(s_{1}^{1}, \ldots, s_{n-1}^{1}\right)$. Then we choose a point $M^{2} \in \Upsilon$ so that it satisfies the conditoin $C_{1} b<\min \left|M^{2}-M^{1}\right|<C_{2} b$, where $b>0, C_{i}$ are positive constant. In the vicinity 
of point $M^{2}$ we also introduce orthogonal coordinates $s^{2}=\left(s_{1}^{2}, \ldots, s_{n-1}^{2}\right)$. Proceeding in the same way, we choose a set of points $M^{p}$ satisfying the condition $C_{1} b<\min _{j \neq p}\left|M^{j}-M^{p}\right|<C_{2} b$ and to introduce the associated orthogonal coordinates $s^{p}=\left(s_{1}^{p}, \ldots, s_{n-1}^{p}\right)$. We denote $Q_{p}:=$ $\left\{x: 0<s_{i}^{p}<b, i=1, \ldots, n-1, \tau=0\right\}$. By the boundedness of Jacobians we can choose $b>0$ so that the inclusion $\bigcup_{p} Q_{p} \supset \Upsilon$ holds true.

We choose a partition of the unity $1=\sum_{p} \widetilde{\chi}_{p}(x)$ such that for each of functions $\widetilde{\chi}_{p}$ the inequality $0 \leqslant\left\|\widetilde{\chi}_{p}\right\|_{C^{2}\left(\operatorname{supp} \widetilde{\chi}_{p}\right)} \leqslant C$ holds, where constant $C$ is independent of $p$. We shall assume that the support of each cut-off functions is contained in cube $Q_{p}$. Let $\widetilde{J}_{p}$ be the Jacobian of the passage from variables $x$ to variables $s^{p}$ satisfying the inequality $\left|\widetilde{J}_{p}\right| \leqslant C$ in $Q_{p}$, where constant $C$ is independent of $p$. As the result of such change we make one-to-one correspondence between the boundary $\Upsilon$ and $\bigcup_{p} Q_{p}$. Each point $x \in \bigcup_{p} Q_{p}$ belongs to a finite amount of supports and this amount is bounded uniformly in $p$. We denote $\beta_{\varepsilon}:=K-K_{0} G_{n} \alpha_{\varepsilon}$. In accordance with the definition of function $\alpha_{\varepsilon}$ we obtain

$$
K_{3}^{\varepsilon}=\left(\beta_{\varepsilon} u_{0}, \varphi_{\varepsilon}\right)_{L_{2}(\Upsilon)}=\sum_{p} \int_{\operatorname{supp} \widetilde{\chi}_{p}} \beta_{\varepsilon} \widetilde{\chi}_{p} \widetilde{J}_{p} u_{0} \varphi_{\varepsilon} d s^{p}=\sum_{p} \int_{Q_{p}} \beta_{\varepsilon} \widetilde{\chi}_{p} \widetilde{J}_{p} u_{0} \varphi_{\varepsilon} d s^{p} .
$$

We expand function $\beta_{\varepsilon}$ into the Fourier series in $Q_{p}$ :

$$
\beta_{\varepsilon}\left(s^{p}\right)=\sum_{q \in \mathbb{Z}^{n-1}} C_{q} \mathrm{e}^{\frac{2 \pi \mathrm{i}}{b} q \cdot s^{p}}, \quad \sum_{q \in \mathbb{Z}^{n-1}}\left|C_{q}\right|^{2} \leqslant\left\|\beta_{\varepsilon}\right\|_{L_{2}\left(Q_{p}\right)}^{2}<\infty .
$$

We introduce the function $U_{p}\left(s^{p}, \tau\right)=-\sum_{q \neq 0} C_{q} \frac{\mathrm{e}^{\frac{2 \pi \mathrm{i}}{b} q \cdot s^{p}-|q| \tau}}{|q|}$. It is easy to check that the series converges $W_{2}^{1}\left(\Sigma_{p}\right), \Sigma_{p}:=\left\{z^{p}: 0<\tau<a, s^{p} \in Q_{p}\right\}, z^{p}=\left(s^{p}, \tau\right), a>0$ is an arbitrary constant. Function $U_{p}$ is the generalized solution to the boundary value problem

$$
\Delta_{z^{p}} U_{p}=0, \quad z^{p} \in \Sigma_{p}, \quad \frac{\partial U_{p}}{\partial \nu}=\widetilde{\beta}_{\varepsilon}, \quad s^{p} \in Q_{p},
$$

subject to the homogeneous Neumann condition on the lateral sides of $\Sigma_{p}$. Here

$$
\widetilde{\beta}_{\varepsilon}:=\beta_{\varepsilon}-\left\langle\beta_{\varepsilon}\right\rangle, \quad\left\langle\beta_{\varepsilon}\right\rangle:=\frac{1}{\left|Q_{p}\right|} \int_{Q_{p}} \beta_{\varepsilon} d s .
$$

Let $\chi_{3}$ be an infinitely differentiable cut-off function being equal to one as $\tau<\frac{a}{3}$ and vanishing as $\tau>\frac{a}{2}$. We integrate by parts

$$
0=-\int_{\Sigma_{p}} \widetilde{\chi}_{p} \widetilde{J}_{p} \chi_{3} u_{0} \varphi_{\varepsilon} \Delta_{z^{p}} U_{p} d z^{p}=-\int_{Q_{p}} \widetilde{\chi}_{p} \widetilde{J}_{p} u_{0} \varphi_{\varepsilon} \frac{\partial U_{p}}{\partial \nu} d s^{p}+\int_{\Sigma_{p}} \nabla_{z^{p}} U_{p} \cdot \nabla_{z^{p}}\left(\widetilde{\chi}_{p} \widetilde{J}_{p} \chi_{3} u_{0} \varphi_{\varepsilon}\right) d z^{p} .
$$

Let us estimate the norm of $\nabla_{z^{p}} U_{p}$ in $L_{2}\left(\Sigma_{p}\right)$ :

$$
\left\|\nabla_{z^{p}} U_{p}\right\|_{L_{2}\left(\Sigma_{p}\right)}^{2}=2 \sum_{q \neq 0}\left|C_{q}\right|^{2} \int_{0}^{a} \mathrm{e}^{-2|q| \tau} d \tau \leqslant C \sum_{q \neq 0} \frac{\left|C_{q}\right|^{2}}{|q|}
$$


In accordance with the definition of functions $U_{p}$, the identities in (3.14), the last inequality, (3.13), (3.15) and the boundedness of Jacobians we get

$$
\begin{aligned}
\left|K_{3}^{\varepsilon}\right| & \leqslant C\left\|u_{0}\right\|_{W_{2}^{2}(\Omega)}\left\|\varphi_{\varepsilon}\right\|_{W_{2}^{1}(\Omega)}\left(\left(\sum_{q \neq 0} \frac{\left|C_{q}\right|^{2}}{|q|}\right)^{\frac{1}{2}}+\left|\left\langle\beta_{\varepsilon}\right\rangle\right|\right) \\
& \leqslant C\left(\sum_{q \in \mathbb{Z}^{n-1}} \frac{\left|C_{q}\right|^{2}}{|q|+1}\right)^{\frac{1}{2}}\left\|u_{0}\right\|_{W_{2}^{2}(\Omega)}\left\|\varphi_{\varepsilon}\right\|_{W_{2}^{1}(\Omega)} .
\end{aligned}
$$

It remains to apply inequality (2.9) to complete the proof.

It follows from the obtained estimates for $K_{1}^{\varepsilon}, K_{2}^{\varepsilon}$ and $K_{3}^{\varepsilon}$ that

$$
\left|g_{\varepsilon}\left(W_{\varepsilon} u_{0}, \varphi_{\varepsilon}\right)+\left(K u_{0}, \varphi_{\varepsilon}\right)_{L_{2}(\Upsilon)}\right| \leqslant C\left(\varepsilon^{\frac{1}{2}}+\mu+\kappa(\varepsilon)\right)\|f\|_{L_{2}(\Omega)}\left\|\varphi_{\varepsilon}\right\|_{W_{2}^{1}(\Omega)} .
$$

By Lemmata 3.2, 3.3 and 3.4 we have

$$
\begin{aligned}
& \left\|\varphi_{\varepsilon}\right\|_{W_{2}^{1}(\Omega)}^{2} \leqslant C\left(\varepsilon^{\frac{1}{2}}+\mu+\kappa(\varepsilon)\right)\|f\|_{L_{2}(\Omega)}\left\|\varphi_{\varepsilon}\right\|_{W_{2}^{1}(\Omega)}, \\
& \left\|\varphi_{\varepsilon}\right\|_{W_{2}^{1}(\Omega)} \leqslant C\left(\varepsilon^{\frac{1}{2}}+\mu+\kappa(\varepsilon)\right)\|f\|_{L_{2}(\Omega)} .
\end{aligned}
$$

By two last inequalities and the first inequality in Lemma 3.4 we obtain:

$$
\left\|u_{\varepsilon}-u_{0}\right\|_{L_{2}(\Omega)} \leqslant C\left(\varepsilon^{\frac{1}{2}}+\mu+\kappa(\varepsilon)\right)\|f\|_{L_{2}(\Omega)} .
$$

Together with (3.16) it completes the proof of the theorem.

We recall that in the proof we employed the assumption that $\lambda$ has a non-zero imaginary part. For such $\lambda$, as two last inequalities say, the resolvent of operator $\mathcal{H}_{\varepsilon}$ converge to the resolvent of operator $\mathcal{H}_{0}$ as $\varepsilon \rightarrow 0$ in the operator norm. Then by such convergence, the spectrum of the perturbed operator converges to the spectrum of the limiting operator. Then by such convergence, the spectrum of the perturbed operator converges to the spectrum of the limiting operator. This is why, if $\lambda$ belongs to compact set $D$ and this compact set does not intersect with the spectrum of operator $\mathcal{H}_{0}$, then $\lambda$ is uniformly separated from the spectrum of operator $\mathcal{H}_{\varepsilon}$ for sufficiently small $\varepsilon$. Then in accordance with [28, Ch. 5, Sect. 5.3, Ineq. (5.3)] estimate (3.6) holds true. In other estimate the fact that the imaginary part of $\lambda$ is non-zero was not used and this is they remain true. Theorem 2.1 is proven.

\section{Formal CONSTRUCTION OF ASYMPTOTICS}

In the present section we prove 2.2, In what follows by $\mathcal{L}$ we mean differential expression (2.4), while function $u_{\varepsilon}$ will be treated as the solution to the boundary value problem

$$
\begin{gathered}
(\mathcal{L}-\lambda) u_{\varepsilon}=f, \quad x \in \Omega, \\
u_{\varepsilon}=0, \quad x \in \gamma_{\varepsilon}, \quad\left(\frac{\partial}{\partial \nu}+a\right) u_{\varepsilon}=0, \quad x \in \Gamma_{\varepsilon} \cup \Xi .
\end{gathered}
$$

In this part of the work we make only formal construction for the solution to this problem. The justification of the asymptotics is made by analogy with the justification of the asymptotics made in [14, Sect. 7].

We work [14], the asymptotics to the solution of problem (4.1), (4.2) was constructed as (2.15). In our case the structure of series (2.16), (2.17), (2.18) is different. Namely, in [14], each of these infinite series was power in $\varepsilon, \eta, \frac{\eta^{n-2}}{\varepsilon}$ and $\ln \eta$, while in our case it is power only in $\varepsilon, \eta$ and $\ln \eta$. Moreover, in the present work each of the coefficients in these series depend on parameter $\mu$. Of course, we can let $\varepsilon=\eta^{n-2}$ in the asymptotic series in [14, Thm. 2.2], 
which, as it was said, were power in $\frac{\eta^{n-2}}{\varepsilon}$. As a result, we shall have infinite series, in which the quotient $\frac{\eta^{n-2}}{\varepsilon}$ is constant, and then we can apply the obtained series for our case. However, these series are no more asymptotic. This is why we shall construct the asymptotics of the solution to problem (4.1), (4.2) as (2.15), (2.16), (2.17), (2.18).

We construct the formal asymptotic expansion for the solution of problem (4.1), (4.2) on the basis of boundary layer method [30], the multiscale method [31] and the method of matching asymptotic expansions [32. Outside small neighbourhoods of boundary $\gamma_{\varepsilon}$, we shall seek function $u_{\varepsilon}$ as the sum of external expansion and the boundary layer:

$$
u_{\varepsilon}(x, \mu, \eta)=u_{\varepsilon}^{\mathrm{ex}}(x, \eta, \mu)+u_{\varepsilon}^{\mathrm{bl}}\left(x^{\prime}, \eta, \mu, \xi\right) .
$$

We shall construct the external expansion $u_{\varepsilon}^{\mathrm{ex}}(x, \mu, \eta)$ in the form of (2.16). It is easy to see that function $u_{\varepsilon}^{\mathrm{ex}}(x, \mu, \eta)$ solves equation (4.1) and satisfies boundary condition on $\Xi$, but it does not satisfy the required boundary conditions on $\gamma_{\varepsilon}$ and $\Gamma_{\varepsilon}$. This is why in the neighbourhood $\Upsilon:=\left\{x: x_{n}=0\right\}$ we construct the boundary layer $u_{\varepsilon}^{\mathrm{bl}}\left(\xi, x^{\prime}, \mu\right)$ as (2.17) in order to satisfy the boundary conditions in (4.2), where $\xi=\left(\xi^{\prime}, \xi_{n}\right)=\left(x^{\prime} \varepsilon^{-1}, x_{n} \varepsilon^{-1}\right)$. In order to construct the boundary layer, we shall employ the boundary layer method and the multiscale method. The sum of external expansion and boundary layer does not satisfy the Dirichlet condition on $\gamma_{\varepsilon}$. This is why in the neighbourhoods of points $M_{\varepsilon}^{k}$ we introduce the rescaled coordinates $\zeta=\left(\xi^{\prime \prime} \eta^{-1}, \xi_{n} \eta^{-1}\right)=\left(\left(x^{\prime}-M_{\varepsilon}^{k}\right)(\varepsilon \eta)^{-1}, x_{n}(\varepsilon \eta)^{-1}\right)$, and we construct the asymptotics of function $u_{\varepsilon}$ on the basis the method of matching asymptotic expansion in small neighbourhoods of points $M_{\varepsilon}^{k}$ in the form of (2.18). The aim of the formal construction is to determined functions $u_{q_{\varepsilon}, q_{\eta}, q_{l}}$, $v_{q_{\varepsilon}, q_{\eta}, q_{l}}$ and $w_{q_{\varepsilon}, q_{\eta}, q_{l}}$.

Although the structure of the series in [14, Thm. 2.2] differs from ours, the problems for the coefficients of the boundary layer, external and internal expansions will be similar to the corresponding problems in Sections 4,5 and 6 in [14]. This is why in this part of the work we write out only the problems for the coefficients. The solvability of these problems can be proved in the same way as in [14]. All the lemmata in this part of the work will be given without the proofs. They can be proved in the same way as in [14].

We begin with the external expansion. Substituting expansion (2.16) into (4.1), (4.2) and equating the coefficients at the like powers of $\varepsilon, \eta$ and $\ln \eta$, we obtain the equations for the coefficients of the external expansion:

$$
\begin{gathered}
(\mathcal{L}-\lambda) u_{0,0,0}=f, \quad x \in \Omega, \\
(\mathcal{L}-\lambda) u_{q_{\varepsilon}, q_{\eta}, q_{l}}=0, \quad x \in \Omega, \quad\left(q_{\varepsilon}, q_{\eta}, q_{l}\right) \neq(0,0,0),
\end{gathered}
$$

as well as the boundary conditions for $u_{q_{\varepsilon}, q_{\eta}, q_{l}}$ :

$$
\left(\frac{\partial}{\partial \nu}+a\right) u_{q_{\varepsilon}, q_{\eta}, q_{l}}=0, \quad x \in \Xi
$$

The boundary conditions on $\Upsilon$ for the coefficients of the external expansion will be determined later while constructing the internal expansion.

We expand coefficients $A_{i j}(x), A_{j}(x)$ and $A_{0}(x)$ into the Taylor series as $x_{n} \rightarrow 0$, and then we shall make the change $x_{n}=\varepsilon \xi_{n}$ :

$$
\begin{aligned}
& A_{i j}(x)=\sum_{k=0}^{\infty} x_{n}^{k} \frac{\partial^{k} A_{i j}}{\partial x_{n}^{k}}\left(x^{\prime}, 0\right)=\sum_{k=0}^{\infty}\left(\varepsilon \xi_{n}\right)^{k} \frac{\partial^{k} A_{i j}}{\partial x_{n}^{k}}\left(x^{\prime}, 0\right)=\delta_{i j}+\sum_{k=1}^{\infty}\left(\varepsilon \xi_{n}\right)^{k} \frac{\partial^{k} A_{i j}}{\partial x_{n}^{k}}\left(x^{\prime}, 0\right), \\
& A_{p}(x)=\sum_{k=0}^{\infty} x_{n}^{k} \frac{\partial^{k} A_{p}}{\partial x_{n}^{k}}\left(x^{\prime}, 0\right)=\sum_{k=0}^{\infty}\left(\varepsilon \xi_{n}\right)^{k} \frac{\partial^{k} A_{p}}{\partial x_{n}^{k}}\left(x^{\prime}, 0\right), \quad p=0, \ldots, n,
\end{aligned}
$$


where $\delta_{i j}$ is the Kronecker delta. The convergence of these series is not assumed, the series are treated as asymptotic ones.

Following the boundary layer method, we postulate that the sum of functions $u_{\varepsilon}^{\mathrm{ex}}$ and $u_{\varepsilon}^{\mathrm{bl}}$ satisfies required homogeneous condition everywhere on $\Upsilon$ except points $M_{\varepsilon}^{k}$ :

$$
\left(\frac{\partial}{\partial \nu}+a\right)\left(u_{\varepsilon}^{\mathrm{ex}}+u_{\varepsilon}^{\mathrm{bl}}\right)=0, \quad x \in \mathbb{R}^{n-1}, \quad x \neq M_{\varepsilon}^{k} .
$$

We substitute (4.6), (4.7) into (4.2), rewrite the second term of the last identity in variables $\xi$, replace functions $u_{\varepsilon}^{\mathrm{ex}}$ and $u_{\varepsilon}^{\mathrm{bl}}$ by the right hand sides of identities (2.16) and (2.17), respectively. In the obtained formula we calculate the coefficients at the like powers of $\varepsilon, \eta$ and $\ln \eta$ and equate these coefficients with zero. As the result we obtain the boundary conditions for functions $v_{q_{\varepsilon}, q_{\eta}, q_{l}}$ :

$$
\begin{aligned}
& \frac{\partial v_{q_{\varepsilon}, q_{\eta}, q_{l}}}{\partial \xi_{n}}=\Psi_{q_{\varepsilon}-1, q_{\eta}, q_{l}}, \quad \xi \in \mathbb{R}^{n-1}, \quad \xi \neq M^{k}, \\
& \Psi_{q_{\varepsilon}, q_{\eta}, q_{l}}:=\Psi_{q_{\varepsilon}, q_{\eta}, q_{l}}\left(x^{\prime}, \mu\right)=\left.\left(\frac{\partial}{\partial \nu}+a\right) u_{q_{\varepsilon}, q_{\eta}, q_{l}}\right|_{x_{n}=0},
\end{aligned}
$$

$M^{k}$ are the images of points $M_{\varepsilon}^{k}$ under the mapping $x \mapsto \varepsilon^{-1} \xi$ and these points are of the form $M^{k}=\left(a_{1} k_{1}, \ldots, a_{n-1} k_{n-1}\right)$.

We substitute expansion (2.17) and series (4.6), (4.7) into equation (4.1), pass to variables $\xi$ and in the obtained identity we write out the terms with the like powers of $\varepsilon, \eta$ and $\ln \eta$. Then for functions $v_{q_{\varepsilon}, q_{\eta}, q_{l}}$ we obtain the equation

$$
\begin{aligned}
& \Delta_{\xi} v_{q_{\varepsilon}, q_{\eta}, q_{l}}=F_{q_{\varepsilon}, q_{\eta}, q_{l}}, \quad \xi_{n}>0, \\
& F_{q_{\varepsilon}, q_{\eta}, q_{l}}:=\sum_{l=1}^{q_{\varepsilon}-1} \sum_{i, j=1}^{n}\left[\left(2 \xi_{n}^{l} A_{i j}^{l, 0} \frac{\partial^{2}}{\partial \xi_{i} \partial \xi_{j}}-\xi_{n}^{l-1} B_{i j}^{l-1} \frac{\partial}{\partial \xi_{j}}\right) v_{q_{\varepsilon}-l, q_{\eta}, q_{l}}\right. \\
& +\xi_{n}^{l-1}\left(2 A_{i j}^{l, 0}\left(\xi_{n} \rho \frac{\partial}{\partial \xi_{i}}-\frac{\partial^{2}}{\partial \xi_{i} \partial x_{j}}\right)+B_{i j}^{l}\left(\rho-\frac{\partial}{\partial x_{j}}\right)+A_{j}^{0, l}\right) v_{q_{\varepsilon}-(l+1), q_{\eta}, q_{l}} \\
& +\left(\xi_{n}^{l}\left(B_{i j}^{l-1} \frac{\partial \rho}{\partial x_{j}}+A_{i j}^{l, 1}\left(\rho^{2}-2 \frac{\partial \rho}{\partial x_{j}} \frac{\partial}{\partial \xi_{i}}\right)\right)-2 \xi_{n}^{l-1} A_{i j}^{l, 0} \frac{\partial}{\partial x_{j}} \rho\right) v_{q_{\varepsilon}-(l+2), q_{\eta}, q_{l}} \\
& -2 \xi_{n}^{l} A_{i j}^{l, 0}\left(\rho \frac{\partial \rho}{\partial x_{j}}+\xi_{n}\left(\frac{\partial^{2} \rho}{\partial x_{i} \partial x_{j}}+\frac{\partial \rho}{\partial x_{i}} \frac{\partial}{\partial x_{j}}\right)\right) v_{q_{\varepsilon}-(l+3), q_{\eta}, q_{l}} \\
& +\left(\xi_{n}^{l+2} A_{i j}^{l, 0} \frac{\partial \rho}{\partial x_{i}} \frac{\partial \rho}{\partial x_{j}}\right) v_{q_{\varepsilon}-(l+4), q_{\eta}, q_{l}}+\left(2 \rho \frac{\partial}{\partial \xi_{n}}-\frac{\partial^{2}}{\partial \xi_{j} \partial x_{j}}\right) v_{q_{\varepsilon}-1, q_{\eta}, q_{l}} \\
& +\left(\rho^{2}+\frac{\partial^{2}}{\partial x_{j}^{2}}-\xi_{n} \frac{\partial \rho}{\partial x_{j}} \frac{\partial}{\partial \xi_{j}}-\lambda\right) v_{q_{\varepsilon}-2, q_{\eta}, q_{l}}+\xi_{n}\left(\frac{\partial^{2} \rho}{\partial x_{j}^{2}}+2 \frac{\partial \rho}{\partial x_{j}}\right) v_{q_{\varepsilon}-3, q_{\eta}, q_{l}} \\
& \left.+\left(\xi_{n} \frac{\partial \rho}{\partial x_{j}}\right)^{2} v_{q_{\varepsilon}-4, q_{\eta}, q_{l}}\right] \\
& A_{q}^{l}:=\frac{\partial^{l} A_{q}}{\partial x_{n}^{l}}, A_{i j}^{l, p}:=\frac{\partial^{l+p} A_{i j}}{\partial x_{n}^{l} \partial x_{i}^{p}}, A_{j}^{0, l}:=A_{0}^{l}-\bar{A}_{j}^{l+1}, B_{i j}^{l}:=A_{j}^{l}-\bar{A}_{j}^{l}-A_{i j}^{l, 1}, p=0,1, q \geqslant 0 .
\end{aligned}
$$

Here the derivatives of coefficients $A_{i j}$ and $A_{q}$ are taken at points $\left(x^{\prime}, 0\right)$.

Thus, for functions $v_{q_{\varepsilon}, q_{\eta}, q_{l}}$, we obtain problems (4.8), (4.10) with a periodic structure in variables $\xi^{\prime}$. In accordance with boundary layer method, these functions are to be exponentially decaying as $\xi_{n} \rightarrow+\infty$. In constructing the boundary layer, we shall also employ the multiscale method. In addition we shall assume that functions $v_{q_{\varepsilon}, q_{\eta}, q_{l}}$ are $\square$-periodic in variables $\xi^{\prime}$, where 
$\square:=\left\{\xi:-\frac{a_{i}}{2}<\xi_{i}<\frac{a_{i}}{2}, \xi_{n}=0\right\}$. Then the original boundary value problem is reduced to the problem in $\Pi=\left\{\xi:-\frac{a_{i}}{2}<\xi_{i}<\frac{a_{i}}{2}, \xi_{n}>0\right\}$ with periodic boundary conditions on the lateral sides of $\Pi$. Hereinafter, the solutions to the problems considered in $\Pi$ will be assumed to satisfy periodic boundary conditions on the lateral sides of $\Pi$.

We denote: $\square:=\left\{\xi:-\frac{a_{i}}{2}<\xi_{i}<\frac{a_{i}}{2}, \xi_{n}=0\right\} \backslash\{0\}$. We treat the solutions to boundary value problem (4.8), (4.10) in the generalized sense. Namely, the solution are functions in space $W_{2}^{1}(\Pi)$ satisfying the integral identity

$$
-\left(\nabla_{\xi} v_{q_{\varepsilon}, q_{\eta}, q_{l}}, \nabla_{\xi} \varpi\right)_{L_{2}(\Pi)}+\left(\Psi_{q_{\varepsilon}-1, q_{\eta}, q_{l}}, \varpi\right)_{L_{2}(\varpi)}=\left(F_{q_{\varepsilon}, q_{\eta}, q_{l}}, \varpi\right)_{L_{2}(\Pi)}
$$

for all functions $\varpi \in C^{\infty}\left(\bar{\Pi}_{R}\right)$, where $\Pi_{R}=\left\{\xi:-\frac{a_{i}}{2}<\xi_{i}<\frac{a_{i}}{2}, 0<\xi_{n}<R\right\}, R=$ const $>0$, functions $\varpi$ should vanish as $\xi_{n}>R$ and satisfy periodic boundary conditions on the lateral sides of $\Pi$.

In order to solve problems (4.8), (4.10) subject to periodic boundary conditions on the lateral sides of $\Pi$, we introduce the auxiliary problem

$$
\Delta_{\zeta} X=0, \quad \xi \in \Pi, \quad \frac{\partial X}{\partial \xi_{n}}=1, \quad \xi \in \bullet .
$$

The solution to this problem exists, it is unique, satisfies periodic boundary conditions on the lateral sides of $\Pi$ and decays exponentially as $\xi_{n} \rightarrow+\infty$ (see [14, Lm. 5.2]). In a small neighbourhood of zero, the identity $X=T_{0}|\xi|^{-n+2}+\widetilde{X}$ holds true, where constant $T_{0}$, we recall, has the form $T_{0}:=-\frac{|\square|}{\left|C_{n}\right|}$, while $\widetilde{X}$ is an infinitely differentiable function in the same neighbourhood.

We introduce spherical coordinates $(r, \theta)$, where $r=|\xi|$, and $\theta$ are coordinates on the unit half-sphere in $\mathbb{R}^{n}$. We denote $\Delta_{\theta}^{N}$ the Laplace-Beltrami operator on the unit $(n-1)$ dimensional half-sphere $S_{1}^{n-1}$ located in the half-space $\left\{\xi: \xi_{n}>0\right\}$ subject to Neumann condition on the edge of the sphere.

By [33. Ch. 5, Sect. 2], the eigenvalues of operator $\Delta_{\theta}^{N}$ are numbers $s(s+n-2)$, where $s$ is an integer positive number. These eigenvalues are simple. We denote by $Y_{s}(\theta)$ the eigenvalues of operator $\Delta_{\theta}^{N}$ corresponding to the eigenvalues of $s(s+n-2)$. These eigenfunctions are chosen orthonormalized in $L_{2}\left(S_{1}^{n-1}\right)$.

In accordance with [33, Ch. 5, Sect. 2], function $Y_{s}(\theta)\left(|\xi|^{-n+2-s}+|\xi|^{s}\right)$ is the solution to the Laplace equation in the small neighbourhood of zero, satisfying Neumann condition on the boundary of this neighbourhood as $\xi_{n}=0$. In [14], the following Lemma 5.3 was proved.

Lemma 4.1. There exist functions $X_{s}$ being solutions to the problem

$$
\Delta X_{s}=0, \quad \xi \in \Pi, \quad \frac{\partial X_{s}}{\partial \xi_{n}}=0, \quad \xi \in \bullet,
$$

satisfying periodic boundary conditions on the lateral sides of $\Pi$, having the asymptotics

$$
X_{s}(\xi)=Y_{s}(\theta)\left(|\xi|^{-n+2-s}+|\xi|^{s}\right)+\sum_{\alpha \in \mathbb{Z}_{+}^{n}} G_{\alpha} \xi^{\alpha}
$$

in the small neighbourhood of zero, where $s \geqslant 0, \xi^{\alpha}:=\xi_{1}^{\alpha_{1}} \xi_{2}^{\alpha_{2}} \ldots \xi_{n}^{\alpha_{n}}$, and $G_{\alpha}$ are some constants.

As it was said, the solvability of problems (4.8), (4.10) with periodic boundary conditions on the lateral sides of $\Pi$ can be prove in the same way as in Sections 4 and 5 in [14]. By analogy with Section 5 in [14], one can also prove that tcoefficients $v_{q_{\varepsilon}, q_{\eta}, q_{l}}$ of the boundary layer are of 
the form:

$$
v_{q_{\varepsilon}, q_{\eta}, q_{l}}\left(x^{\prime}, \mu, \xi\right)=v_{q_{\varepsilon}, q_{\eta}, q_{l}}^{0}\left(x^{\prime}, \mu, \xi\right)+\Psi_{q_{\varepsilon}-1, q_{\eta}, q_{l}}\left(x^{\prime}, \mu\right) X(\xi)+\sum_{i=0}^{q_{\eta}-q_{l}-1} A_{i}^{q_{\varepsilon}, q_{\eta}, q_{l}}\left(x^{\prime}, \mu\right) X_{i}(\xi) .
$$

Coefficients $A_{i}^{q_{\varepsilon}, q_{\eta}, q_{l}}\left(x^{\prime}, \mu\right)$ will be determined in what follows. The obtained solutions in a small neighbourhood of zero have the asymptotics:

$$
\begin{aligned}
v_{q_{\varepsilon}, q_{\eta}, q_{l}}\left(x^{\prime}, \mu, \xi\right)= & \Psi_{q_{\varepsilon}-1, q_{\eta}, q_{l}}\left(x^{\prime}, \mu\right) T_{0}|\xi|^{-n+2}+B^{q_{\varepsilon}, q_{\eta}, q_{l}}\left(x^{\prime}, \mu\right)+\sum_{i=0}^{\infty} Y_{2, i, 0}^{q_{\varepsilon}, q_{\eta}, q_{l}}\left(x^{\prime}, \mu, \theta\right)|\xi|^{i} \\
& +\sum_{i=0}^{q_{\eta}+q_{\varepsilon}-q_{l}-1} Y_{1, i, 0}^{q_{\varepsilon}, q_{\eta}, q_{l}}\left(x^{\prime}, \mu, \theta\right)|\xi|^{-n+\widehat{K}_{0}-i} \\
& +\bar{\delta}_{q_{l}, Q_{q_{\varepsilon}, q_{\eta}}-1}\left(\sum_{j=1}^{q_{\varepsilon}-q_{l}-1} \sum_{i=0}^{q_{\eta}-2 j+q_{\varepsilon}-1} Y_{1, i, j}^{q_{\varepsilon}, q_{\eta}, q_{l}}\left(x^{\prime}, \mu, \theta\right)|\xi|^{-n+\widehat{K}_{j}-i} \ln ^{j}|\xi|\right. \\
& +\sum_{i=0}^{q_{\varepsilon}-q_{l}-n+1} \sum_{j=1}^{i} Y_{3, i, j}^{q_{\varepsilon}, q_{\eta}, q_{l}}\left(x^{\prime}, \mu, \theta\right)|\xi|^{i} \ln ^{j}|\xi| \\
& \left.+\sum_{j=1}^{q_{\varepsilon}-q_{l}-1} \sum_{i=0}^{\infty} Y_{2, i, j}^{q_{\varepsilon}, q_{\eta}, q_{l}}\left(x^{\prime}, \mu, \theta\right)|\xi|^{i+j+1} \ln ^{j}|\xi|\right)
\end{aligned}
$$

where $\widehat{K}_{j}:=1+q_{\varepsilon}-j-q_{l}+\bar{\delta}_{q_{l}, q_{\varepsilon}-1}, \bar{\delta}_{p, q}:=1-\delta_{p, q}$,

$$
\begin{aligned}
& B^{q_{\varepsilon}, q_{\eta}, q_{l}}\left(x^{\prime}, \mu\right):=\Psi_{q_{\varepsilon}-1, q_{\eta}, q_{l}}\left(x^{\prime}, \mu\right) \widetilde{X}_{0}+\mathbf{A}^{q_{\varepsilon}, q_{\eta}, q_{l}}\left(x^{\prime}, \mu\right), \\
& \mathbf{A}^{q_{\varepsilon}, q_{\eta}, q_{l}}\left(x^{\prime}, \mu\right):=G_{0} \sum_{i=0}^{q_{\eta}-q_{l}-1} A_{i}^{q_{\varepsilon}, q_{\eta}, q_{l}}\left(x^{\prime}, \mu\right) .
\end{aligned}
$$

Here $\widetilde{X}_{0}$ is the leading coefficient in the Taylor series for function $\widetilde{X}$ in a small neighbourhood of zero.

Remark 4.1. Functions $Y_{k, i, j}^{q_{\varepsilon}, q_{\eta}, q_{l}}\left(x^{\prime}, \mu, \theta\right), q_{\varepsilon} \geqslant 1$, satisfy the identity

$$
Y_{k, i, j}^{q_{\varepsilon}, q_{\eta}, q_{l}}\left(x^{\prime}, \mu, \theta\right)=\sum_{m=0}^{M_{1}} \psi_{m}^{q_{\varepsilon}, q_{\eta}, q_{l}}\left(x^{\prime}, \mu\right) Y_{m, k, i, j}(\theta),
$$

where $\psi_{m}^{q_{\varepsilon}, q_{\eta}, q_{l}}$ is a linear combination of functions $u_{q_{\varepsilon}-2, q_{\eta}, q_{l}}$ and the derivatives of these functions, $M_{1}$ is some number depending on the superscripts of functions $\psi_{m}^{q_{\varepsilon}, q_{\eta}, q_{l}}$, while $Y_{m, k, i, j}$ are infinitely differentiable functions on half-sphere $\overline{S_{1}^{n-1}}$ satisfying Neumann condition on its edge. This fact for $q_{\varepsilon}>2$ will be checked while constructing the internal expansion and matching the asymptotics. In particular, for functions $Y_{k, i, j}^{2, q_{\eta}, q_{l}}\left(x^{\prime}, \mu, \theta\right)$, this identity is implied by the asymptotics of functions $v_{1, q_{\eta}, 0}$ in a small neighbourhood and identity (4.10) for right hand sides $F_{2, q_{\eta}, q_{l}}$.

Remark 4.2. While constructing boundary layer, we added some solutions of the homogeneous problem in Lemma 4.1 with arbitrary coefficients to functions $v_{q_{\varepsilon}, q_{\eta}, q_{l}}$. In accordance with Lemma 4.1, this problem has infinitely many solutions. At that, among the existing set of solutions, we added only the above mentioned. Our apriori choice of the added solutions is due to the conditions appearing in matching of internal and external expansions. 
We denote: $\Gamma_{0}:=\left\{\xi: \xi_{n}=0, \xi \neq M^{k}, k \in \mathbb{Z}^{n-1}\right\}, \Pi^{\delta}:=\Pi \cap\{\xi:|\xi|>\delta\}$. Since the coefficients of boundary layer are $\square$-periodic in $\xi^{\prime}$, the solutions to the considered problems in $\Pi$ are continued periodically for the whole half-space $\left\{\xi: \xi_{n}>0\right\}$. By analogy with [14, Lm. 5.5] we prove

Lemma 4.2. Problems (4.8), (4.10) are solvable, functions $v_{q_{\varepsilon}, q_{\eta}, q_{l}}, F_{q_{\varepsilon}, q_{\eta}, q_{l}}$ can be represented as the sums

$$
\begin{aligned}
& v_{q_{\varepsilon}, q_{\eta}, q_{l}}\left(x^{\prime}, \mu, \xi\right)=\sum_{q=1}^{M_{q_{\varepsilon}}^{1}} \varphi_{q_{\varepsilon}, q_{\eta}, q_{l}}^{q}\left(x^{\prime}, \mu\right) v_{q_{\varepsilon}, q_{\eta}, q_{l}}^{q}(\xi), \\
& F_{q_{\varepsilon}, q_{\eta}, q_{l}}\left(x^{\prime}, \mu, \xi\right)=\sum_{q=1}^{M_{q_{\varepsilon}}^{2}} \psi_{q_{\varepsilon}, q_{\eta}, q_{l}}^{q}\left(x^{\prime}, \mu\right) F_{q_{\varepsilon}, q_{\eta}, q_{l}}^{q}(\xi),
\end{aligned}
$$

where $M_{q_{\varepsilon}}^{j}$ are some numbers, $\varphi_{q_{\varepsilon}, q_{\eta}, q_{l}}^{q}, \quad \psi_{q_{\varepsilon}, q_{\eta}, q_{l}}^{q}$ are finite linear combinations of the traces of the coefficients of the external expansion and their derivatives, $v_{q_{\varepsilon}, q_{\eta}, q_{l}}^{q} \in W_{2}^{1}\left(\Pi^{\delta}\right)$, $v_{q_{\varepsilon}, q_{\eta}, q_{l}}^{q} \in C^{\infty}\left(\Gamma_{0} \cup\left\{\xi: \xi_{n}>0\right\}\right), F_{q_{\varepsilon}, q_{\eta}, q_{l}}^{q} \in C^{\infty}\left(\Gamma_{0} \cup\left\{\xi: \xi_{n}>0\right\}\right), F_{q_{\varepsilon}, q_{\eta}, q_{l}}^{q} \in L_{2}\left(\Pi^{\delta}\right)$. Here $\delta>0$ is an arbitrary sufficiently small number.

Formal construction of boundary layer (2.17) is completed.

In view of the construction of the boundary layer functions, Lemma 4.1 and the definition of function $X$, these coefficients have a singularity at zero. Moreover, we recall that in accordance with the definition of functions $v_{q_{\varepsilon}, q_{\eta}, q_{l}}$, the sum of the external expansion and boundary layer does not satisfy Dirichlet condition on $\gamma_{\varepsilon}$. This is why following the method of matching asymptotics expansions, in the vicinities of points $M_{\varepsilon}^{k}$ we construct the internal expansion. First, this expansion controls the required boundary conditions in the vicinity of $\gamma_{\varepsilon}$, and second, it should be matched at infinity with previously constructed formal asymptotic solutions for the coefficients of the boundary layer in a small neighbourhood of points $M^{k}$. Since the functions of boundary layer are $\square$-periodic in variables $\xi^{\prime}$, it is sufficient to match asymptotic expansion in the vicinity of zero. In the vicinities of points $M_{\varepsilon}^{k}$ the internal expansion is constructed in the same way but in term of variables $\zeta^{k}=\left(\left(x^{\prime}-M_{\varepsilon}^{k}\right)(\varepsilon \eta)^{-1}, x_{n}(\varepsilon \eta)^{-1}\right)$.

We expand the coefficients of the external expansion and function $\mathrm{e}^{-\rho\left(x^{\prime}\right) x_{n}}$ in (2.17) into the Taylor series w.r.t. variable $x_{n}$ in a small neighbourhood of zero and then we make the change $x_{n}=\varepsilon \eta \zeta_{n}$ :

$$
\mathrm{e}^{-\rho\left(x^{\prime}\right) x_{n}} u_{q_{\varepsilon}, q_{\eta}, q_{l}}(x, \mu)=\sum_{i=0}^{\infty}\left(\varepsilon \eta \zeta_{n}\right)^{i} \sum_{j=0}^{i} \frac{\left(-\rho\left(x^{\prime}\right)\right)^{i-j}}{j !(i-j) !} \gamma_{q_{\varepsilon}, q_{\eta}, q_{l}}^{j}\left(x^{\prime}, \mu\right)
$$

where we denote

$$
\gamma_{q_{\varepsilon}, q_{\eta}, q_{l}}^{j}:=\frac{\partial^{j} u_{q_{\varepsilon}, q_{\eta}, q_{l}}}{\partial x_{n}^{j}}\left(x^{\prime}, 0\right)
$$

It follows that

$$
u_{q_{\varepsilon}, q_{\eta}, q_{l}}(x, \mu)=\mathrm{e}^{\rho\left(x^{\prime}\right) x_{n}} \sum_{i=0}^{\infty}\left(\varepsilon \eta \zeta_{n}\right)^{i} \sum_{j=0}^{i} \frac{\left(-\rho\left(x^{\prime}\right)\right)^{i-j}}{j !(i-j) !} \gamma_{q_{\varepsilon}, q_{\eta}, q_{l}}^{j}\left(x^{\prime}, \mu, \eta\right) .
$$

We rewrite asymptotics (2.16) and (2.17) in variables $\zeta=\eta^{-1} \xi$ :

$$
u_{\varepsilon}^{\mathrm{ex}}(x, \mu, \eta)+u_{\varepsilon}^{\mathrm{bl}}\left(\xi, x^{\prime}, \eta, \mu\right)=\mathrm{e}^{\rho\left(x^{\prime}\right) x_{n}} \sum_{q_{\varepsilon}, q_{\eta}=0}^{\infty} \sum_{q_{l}=0}^{Q_{q_{\varepsilon}, q_{\eta}}} \varepsilon^{q_{\varepsilon}} \eta^{q_{\eta}} \ln ^{q_{l}} \eta \varphi_{q_{\varepsilon}, q_{\eta}, q_{l}}\left(x^{\prime}, \mu, \zeta\right) .
$$


Here $\varphi_{0, q_{\eta}, 0}$ and $\varphi_{q_{\varepsilon}, 0,0}, q_{\varepsilon}, q_{\eta} \geqslant 0$ are introduced by the identities

$$
\begin{aligned}
\varphi_{0, q_{\eta}, 0}\left(x^{\prime}, \mu, \zeta\right)= & \gamma_{0, q_{\eta}, 0}^{0}\left(x^{\prime}, \mu\right)+\rho_{\varepsilon}\left(\left(\Psi_{0, q_{\eta}, 0}\left(x^{\prime}, \mu\right) T_{0}+A_{0}^{1, q_{\eta}-1,0}\left(x^{\prime}, \mu\right)\right)|\zeta|^{-n+2}\right. \\
& \left.+\sum_{i=0}^{\infty} Y_{1, i, 0}^{1, q_{\eta}+1+i, 0}\left(x^{\prime}, \mu, \theta\right)|\zeta|^{-n+1-i}\right), \\
\varphi_{q_{\varepsilon}, 0,0}\left(x^{\prime}, \mu, \zeta\right)= & \gamma_{q_{\varepsilon}, 0,0}^{0}\left(x^{\prime}, \mu\right)+\bar{\delta}_{q_{\varepsilon}, 0} B^{q_{\varepsilon}, 0,0}\left(x^{\prime}, \mu\right)+\rho_{\varepsilon}\left(\Psi_{q_{\varepsilon}, 0,0}\left(x^{\prime}, \mu\right) T_{0}|\zeta|^{-n+2}\right. \\
& \left.+\sum_{i=0}^{\infty} Y_{1, i, 0}^{q_{\varepsilon}+1, i+1,0}\left(x^{\prime}, \mu, \theta\right)|\zeta|^{-n+1-i}\right),
\end{aligned}
$$

where $\rho_{\varepsilon}:=\frac{\varepsilon}{\eta^{n-2}}, \bar{\delta}_{p, q}:=1-\delta_{p, q}$. The formulae for other $\varphi_{q_{\varepsilon}, q_{\eta}, q_{l}}$ are as follows:

$$
\begin{aligned}
\varphi_{q_{\varepsilon}, q_{\eta}, q_{l}}\left(\zeta, x^{\prime}, \mu\right)= & \sum_{i=0}^{Q_{q_{\varepsilon}, q_{\eta}}} \sum_{j=0}^{i} \frac{\left(-\rho\left(x^{\prime}\right)\right)^{i-j}}{j !(i-j) !} \gamma_{q_{\varepsilon}-i, q_{\eta}-i, q_{l}}^{j}\left(x^{\prime}, \mu\right) \zeta_{n}^{i} \\
& +\rho_{\varepsilon}\left(\left(\Psi_{q_{\varepsilon}, q_{\eta}, q_{l}}\left(x^{\prime}, \mu\right) T_{0}+A_{0}^{q_{\varepsilon}+1, q_{\eta}-1, q_{l}}\left(x^{\prime}, \mu\right)\right)|\zeta|^{-n+2}\right. \\
& \left.+\sum_{j=0}^{Q_{q_{\varepsilon}, q_{\eta}}-q_{l}} \sum_{i=0}^{\infty} Y_{1, i, j}^{q_{\varepsilon}+1, q_{\eta}-\widetilde{K}_{j}+i, q_{l}}\left(x^{\prime}, \mu, \theta\right)|\zeta|^{-n+\widetilde{K}_{j}-i} \ln ^{j}|\zeta|\right) \\
& +\bar{\delta}_{q_{l}, Q_{q_{\varepsilon}, q_{\eta}}} \sum_{j=1}^{Q_{q_{\varepsilon}, q_{\eta}}-q_{l}} \sum_{i=0}^{q_{\eta}-2} Y_{2, i, j}^{q_{\varepsilon}, q_{\eta}-2-i, q_{l}}\left(x^{\prime}, \mu, \theta\right)|\zeta|^{i+2} \ln ^{j}|\zeta|+B^{q_{\varepsilon}, q_{\eta}, q_{l}}\left(x^{\prime}, \mu\right) \\
& +\sum_{i=0}^{q_{\eta}-\left(q_{l}+1\right)} Y_{2, i, 0}^{q_{\varepsilon}, q_{\eta}-i, q_{l}}\left(x^{\prime}, \mu, \theta\right)|\zeta|^{i}+\sum_{j=1}^{P_{1}} \sum_{i=0}^{P_{2}} Y_{6, i, j}^{q_{\varepsilon}, q_{\eta}, q_{l}}\left(x^{\prime}, \mu, \theta\right)|\zeta|^{i} \ln ^{j}|\zeta|,
\end{aligned}
$$

where $Q_{q_{\varepsilon}, q_{\eta}}:=\min \left(q_{\varepsilon}, q_{\eta}\right), q_{\eta}, q_{\varepsilon}, q_{l} \geqslant 0, P_{1}, P_{2}$ are integer numbers depending on $n, \widetilde{K}_{j}:=$ $Q_{q_{\varepsilon}, q_{\eta}}-j+1-q_{l}+\bar{\delta}_{q_{l}, Q_{q_{\varepsilon}, q_{\eta}-1}}$. By the method of matching asymptotic expansions and taking into consideration the coefficient $\mathrm{e}^{\rho\left(x^{\prime}\right) x_{n}}$ in (2.18), the terms of the internal expansions have the following asymptotics at the infinity:

$$
w_{q_{\varepsilon}, q_{\eta}, q_{l}}=\varphi_{q_{\varepsilon}, q_{\eta}, q_{l}}, \quad \zeta \rightarrow \infty, \quad \zeta_{n}>0 .
$$

We introduce spherical coordinates $(\rho, \theta)$, where $\rho=|\zeta|$, and $\theta$ are coordinates in the unit half-sphere $S_{1}^{n-1}$. Then we rewrite functions $\varphi_{q_{\varepsilon}, q_{\eta}, q_{l}}$ as

$$
\begin{aligned}
\varphi_{q_{\varepsilon}, q_{\eta}, q_{l}}\left(\zeta, x^{\prime}, \mu\right)= & T^{q_{\varepsilon}, q_{\eta}, q_{l}}\left(x^{\prime}, \mu\right)+\rho_{\varepsilon}\left(\Psi_{q_{\varepsilon}, q_{\eta}, q_{l}}\left(x^{\prime}, \mu\right) T_{0}+A_{0}^{q_{\varepsilon}+1, q_{\eta}-1, q_{l}}\left(x^{\prime}, \mu\right)\right)|\zeta|^{-n+2} \\
& +\sum_{j=0}^{Q_{q_{\varepsilon}, q_{\eta}}-q_{l}} \sum_{i=0}^{\infty} Y_{4, i, j}^{q_{\varepsilon}, q_{\eta}, q_{l}}\left(x^{\prime}, \mu, \theta\right)|\zeta|^{-n+\widetilde{K}_{j}-i} \ln ^{j}|\zeta| \\
& \sum_{j=1}^{Q_{q_{\varepsilon}, q_{\eta}}-q_{l}} \sum_{i=0}^{q_{\eta}-2} Y_{5, i, j}^{q_{\varepsilon}, q_{\eta}, q_{l}}\left(x^{\prime}, \mu, \theta\right)|\zeta|^{i+2} \ln ^{j}|\zeta| \\
& +\sum_{i=0}^{q_{\eta}-\left(q_{l}+1\right)} Y_{5, i, 0}^{q_{\varepsilon}, q_{\eta}, q_{l}}\left(x^{\prime}, \mu, \theta\right)|\zeta|^{i}+\sum_{j=1}^{P_{1}} \sum_{i=0}^{P_{2}} Y_{6, i, j}^{q_{\varepsilon}, q_{\eta}, q_{l}}\left(x^{\prime}, \mu, \theta\right)|\zeta|^{i} \ln ^{j}|\zeta|,
\end{aligned}
$$


where

$$
T^{q_{\varepsilon}, q_{\eta}, q_{l}}:=\gamma_{q_{\varepsilon}, q_{\eta}, q_{l}}^{0}+\varrho B^{q_{\varepsilon}, q_{\eta}, q_{l}}=\varrho\left(\mathbf{A}^{q_{\varepsilon}, q_{\eta}, q_{l}}+\Psi_{q_{\varepsilon}-1, q_{\eta}, q_{l}} \widetilde{X}_{0}\right)+\gamma_{q_{\varepsilon}, q_{\eta}, q_{l}}^{0}, \varrho:=\varsigma \bar{\delta}_{q_{\varepsilon}, 0} .
$$

Here function $\varsigma$ vanishes as $q_{l}=Q_{q_{\varepsilon}, q_{\eta}} \neq 0$ and is equal to one in other cases, while functions $Y_{k, i, j}^{q_{\varepsilon}, q_{\eta}, q_{l}}$ are defined by the identities

$$
\begin{aligned}
Y_{4, i, j}^{q_{\varepsilon}, q_{\eta}, q_{l}} & :=\frac{1}{\rho_{\varepsilon}} Y_{1, i, j}^{q_{\varepsilon}+1, q_{\eta}-1-\widetilde{K}_{j}+i, q_{l}}, \quad Y_{5, i, j}^{q_{\varepsilon}, q_{\eta}, q_{l}}:=Y_{2, i, j}^{q_{\varepsilon}, q_{\eta}-2-i, q_{l}}, \\
Y_{5, i, 0}^{q_{\varepsilon}, q_{\eta}, q_{l}} & :=Y_{2, i, 0}^{q_{\varepsilon}, q_{\eta}-i, q_{l}}+\sum_{i=1}^{Q_{q_{\varepsilon}, q_{\eta}}} \sum_{j=0}^{i} \frac{(-\rho)^{i-j}}{j !(i-j) !} \gamma_{q_{\varepsilon}-i, q_{\eta}-i, q_{l}}^{j} .
\end{aligned}
$$

Functions $\mathbf{A}^{q_{\varepsilon}, q_{\eta}, q_{l}}$ are defined in (4.13).

Now we write out the problems for the coefficients of the internal expansion. In order to do it, we expand function $f$ into the Taylor series as $x_{n} \rightarrow 0$, make the change $x_{n}=\varepsilon \eta \zeta_{n}$, substitute (2.18) into (4.1), (4.2) and equate the coefficients at the like powers of $\varepsilon, \eta$ and $\ln \eta$. As the result we obtain the equations and boundary conditions for functions $w_{q_{\varepsilon}, q_{\eta}, q_{l}}$ :

$$
\begin{gathered}
\Delta_{\zeta} w_{q_{\varepsilon}, q_{\eta}, q_{l}}=G_{q_{\varepsilon}, q_{\eta}, q_{l}}, \quad \zeta \in S^{+}, \\
w_{q_{\varepsilon}, q_{\eta}, q_{l}}=0, \quad \zeta \in \Gamma_{1}, \quad \frac{\partial w_{q_{\varepsilon}, q_{\eta}, q_{l}}}{\partial \zeta_{n}}=0, \quad \zeta \in \Gamma_{2} .
\end{gathered}
$$

Here $S^{+}=\left\{\zeta: \zeta_{n}>0\right\}, \Gamma_{1}:=\left\{\zeta \in \mathbb{R}^{n}: \zeta^{\prime} \in \gamma, \zeta_{n}=0\right\}, \Gamma_{2}:=\left\{\zeta: \zeta_{n}=0\right\} \backslash \bar{\Gamma}_{1}$,

$$
\begin{aligned}
& G_{q_{\varepsilon}, q_{\eta}, q_{l}}:=\sum_{l=1}^{Q_{q_{\varepsilon}, q_{\eta}}} \sum_{i, j=1}^{n}\left[\left(2 \zeta_{n}^{l} A_{i j}^{l, 0} \frac{\partial^{2}}{\partial \zeta_{i} \partial \zeta_{j}}-\zeta_{n}^{l-1} B_{i j}^{l-1} \frac{\partial}{\partial \zeta_{j}}\right) w_{q_{\varepsilon}-l, q_{\eta}-l, q_{l}}\right. \\
& +\zeta_{n}^{l-1}\left(2 A_{i j}^{l, 0}\left(\zeta_{n} \rho \frac{\partial}{\partial \zeta_{i}}-\frac{\partial^{2}}{\partial \zeta_{i} \partial x_{j}}\right)+B_{i j}^{l}\left(\rho-\frac{\partial}{\partial x_{j}}\right)+A_{j}^{2, l}\right) w_{q_{\varepsilon}-(l+1), q_{\eta}-(l+1), q_{l}} \\
& +\left(\zeta_{n}^{l}\left(B_{i j}^{l-1} \frac{\partial \rho}{\partial x_{j}}+A_{i j}^{l, 1}\left(\rho^{2}-2 \frac{\partial \rho}{\partial x_{j}} \frac{\partial}{\partial \zeta_{i}}\right)\right)-2 \zeta_{n}^{l-1} A_{i j}^{l, 0} \frac{\partial}{\partial x_{j}} \rho\right) w_{q_{\varepsilon}-(l+2), q_{\eta}-(l+2), q_{l}} \\
& -2 \zeta_{n}^{l} A_{i j}^{l, 0}\left(\rho \frac{\partial \rho}{\partial x_{j}}+\zeta_{n}\left(\frac{\partial^{2} \rho}{\partial x_{i} \partial x_{j}}+\frac{\partial \rho}{\partial x_{i}} \frac{\partial}{\partial x_{j}}\right)\right) w_{q_{\varepsilon}-(l+3), q_{\eta}-(l+3), q_{l}} \\
& +\left(\zeta_{n}^{l+2} A_{i j}^{l, 0} \frac{\partial \rho}{\partial x_{i}} \frac{\partial \rho}{\partial x_{j}}\right) w_{q_{\varepsilon}-(l+4), q_{\eta}-(l+4), q_{l}}+\left(2 \rho \frac{\partial}{\partial \zeta_{n}}-\frac{\partial^{2}}{\partial \zeta_{j} \partial x_{j}}\right) w_{q_{\varepsilon}-1, q_{\eta}-1, q_{l}} \\
& +\left(\rho^{2}+\frac{\partial^{2}}{\partial x_{j}^{2}}-\zeta_{n} \frac{\partial \rho}{\partial x_{j}} \frac{\partial}{\partial \zeta_{j}}-\lambda\right) w_{q_{\varepsilon}-2, q_{\eta}-2, q_{l}}+\zeta_{n}\left(\frac{\partial^{2} \rho}{\partial x_{j}^{2}}+2 \frac{\partial \rho}{\partial x_{j}}\right) w_{q_{\varepsilon}-3, q_{\eta}-3, q_{l}} \\
& \left.+\left(\zeta_{n} \frac{\partial \rho}{\partial x_{j}}\right)^{2} w_{q_{\varepsilon}-4, q_{\eta}-4, q_{l}}\right], \quad A_{j}^{2, l}:=A_{j}^{0, l}+\frac{\partial^{l} f}{\partial x_{n}^{l}} .
\end{aligned}
$$

The derivatives of coefficients $A_{i j}$ and $A_{j}$ as well as the derivatives of function $f$ are taken at points $\left(x^{\prime}, 0\right)$. Thus, functions $w_{q_{\varepsilon}, q_{\eta}, q_{l}}$ of internal expansion are solutions to problems (4.23), (4.19). In order to study he solvability of these problems, we shall need auxiliary statements.

We recall that functions $Y_{s}(\theta)$ were introduced in the previous section as eigenfunctions of operator $\Delta_{\theta}^{N}$ associated with the eigenvalues $s(s+n-2)$. In accordance with 33 , Ch. 5, Sect. 2], function $Y_{s}(\theta)\left(|\zeta|^{s}+|\zeta|^{-n+2-s}\right)$ is the solution to Laplace equation at the infinity. In work [14, the following lemma was proven (see Lemma 6.2 in this work).

Lemma 4.3. There exist functions $\Psi_{s}$ solving the problems

$$
\Delta \Psi_{s}=0, \quad \zeta \in S^{+}, \quad \Psi_{s}=0, \quad \zeta \in \Gamma_{1}, \quad \frac{\partial \Psi_{s}}{\partial \zeta_{n}}=0, \quad \zeta \in \Gamma_{2},
$$


having the asymptotics

$$
\Psi_{s}(\zeta)=Y_{s}(\theta)\left(|\zeta|^{s}+|\zeta|^{-n+2-s}\right)+\sum_{\alpha \in \mathbb{Z}_{+}^{n}} U_{\alpha} \zeta^{\alpha}|\zeta|^{-2|\alpha|-n+2}
$$

as $\zeta \rightarrow \infty$, where $s \geqslant 0, U_{\alpha}$ are some constants.

To solve problems (4.23), we shall make use of problem (2.6). In problem (2.6), as set $\omega^{(i)}$ we take $\Gamma_{1}$ and we denote by $Y$ the solution of such changed problem (2.6). In accordance with [14, Lm. 5.4], the solution to this problem exists, unique and has the asymptotics

$$
Y(\zeta)=\sum_{\alpha \in \mathbb{Z}_{+}^{n}} N_{\alpha} \zeta^{\alpha}|\zeta|^{-2|\alpha|-n+2}
$$

as $\zeta \rightarrow \infty$, where $N_{\alpha}$ are some constants. We shall also need the asymptotics of function $Y(\zeta)$

$$
Y(\zeta)=N_{0}|\zeta|^{-n+2}+\sum_{i=0}^{\infty} \widetilde{Z}_{1, i, 0}(\theta)|\zeta|^{-n+1-i}
$$

at infinity. Here $\widetilde{Z}_{k, i, 0}$ are infinitely differentiable functions on the unit half-sphere $S_{1}^{n-1}$ satisfying Neumann condition on its edge. The convergence of this series is not assumed, the series is treated as an asymptotic one.

Solvability of problems (4.23), (4.20) can be proved by analogy with Section 6 in [14]. Then in the same way as in Section 6 in [14], one can show that coefficients $w_{q_{\varepsilon}, q_{\eta}, q_{l}}$ of internal expansion are of the form:

$w_{q_{\varepsilon}, q_{\eta}, q_{l}}\left(x^{\prime}, \mu, \zeta\right)=\mathbf{T}^{q_{\varepsilon}, q_{\eta}, q_{l}}\left(x^{\prime}, \mu\right)(1-Y(\zeta))+\bar{\delta}_{q_{\varepsilon}, 0}\left(\sum_{i=0}^{q_{\eta}-\left(q_{l}+1\right)} C_{i}^{q_{\varepsilon}, q_{\eta}, q_{l}}\left(x^{\prime}, \mu\right) \Psi_{i}(\zeta)+w_{q_{\varepsilon}, q_{\eta}, q_{l}}^{0}\left(x^{\prime}, \mu, \zeta\right)\right)$,

where functions $\mathbf{T}^{q_{\varepsilon}, q_{\eta}, q_{l}}$ are given by the formulae:

$$
\mathbf{T}^{q_{\varepsilon}, q_{\eta}, q_{l}}:=T^{q_{\varepsilon}, q_{\eta}, q_{l}}-\bar{\delta}_{q_{\varepsilon}, 0} C_{0}^{q_{\varepsilon}, q_{\eta}, q_{l}},
$$

while coefficients $C_{i}^{q_{\varepsilon}, q_{\eta}, q_{l}}\left(x^{\prime}, \mu\right)$ will be determined later. The obtained solutions have the following asymptotics

$$
\begin{aligned}
w_{q_{\varepsilon}, q_{\eta}, q_{l}}\left(x^{\prime}, \mu, \zeta\right)= & T^{q_{\varepsilon}, q_{\eta}, q_{l}}\left(x^{\prime}, \mu\right)+\left.\mathbf{B}^{q_{\varepsilon}, q_{\eta}, q_{l}}\left(x^{\prime}, \mu\right) \zeta\right|^{-n+2}+\sum_{i=0}^{q_{\eta}-\left(q_{l}+1\right)} Z_{2, i, 0}^{q_{\varepsilon}, q_{\eta}, q_{l}}\left(x^{\prime}, \mu, \theta\right)|\zeta|^{i} \\
& +\sum_{j=0}^{Q_{q_{\varepsilon}, q_{\eta}}-q_{l}} \sum_{i=0}^{\infty} Z_{1, i, j}^{q_{\varepsilon}, q_{\eta}, q_{l}}\left(x^{\prime}, \mu, \theta\right)|\zeta|^{-n+\widetilde{K}_{j}-i} \ln ^{j}|\zeta| \\
& +\sum_{j=1}^{Q_{q_{\varepsilon}, q_{\eta}}-q_{l}} \sum_{i=0}^{q_{\eta}-2} Z_{2, i, j}^{q_{\varepsilon}, q_{\eta}, q_{l}}\left(x^{\prime}, \mu, \theta\right)|\zeta|^{i+2} \ln ^{j}|\zeta| \\
& +\sum_{i=0}^{Q_{q_{\varepsilon}, q_{\eta}}-q_{l}-n+2} \sum_{j=1}^{i}{ }^{\left.\frac{i}{n-1}\right]+1} Z_{3, i, j}^{q_{\varepsilon}, q_{\eta}, q_{l}}\left(x^{\prime}, \mu, \theta\right)|\zeta|^{i} \ln ^{j}|\zeta|,
\end{aligned}
$$

at infinity, where $\mathbf{B}^{q_{\varepsilon}, q_{\eta}, q_{l}}$ are of the form:

$$
\mathbf{B}^{q_{\varepsilon}, q_{\eta}, q_{l}}:=-T^{q_{\varepsilon}, q_{\eta}, q_{l}} N_{0}+\bar{\delta}_{q_{\varepsilon}, 0} \mathbf{C}^{q_{\varepsilon}, q_{\eta}, q_{l}}, \quad \mathbf{C}^{q_{\varepsilon}, q_{\eta}, q_{l}}:=C_{0}^{q_{\varepsilon}, q_{\eta}, q_{l}}+U_{0} \sum_{i=0}^{q_{\eta}-\left(q_{l}+1\right)} C_{i}^{q_{\varepsilon}, q_{\eta}, q_{l}} .
$$


Remark 4.3. Functions $Z_{k, i, j}^{q_{\varepsilon}, q_{\eta}, q_{l}}\left(x^{\prime}, \mu, \theta\right)$ satisfy the identity:

$$
Z_{k, i, j}^{q_{\varepsilon}, q_{\eta}, q_{l}}\left(x^{\prime}, \mu, \theta\right)=\sum_{m=0}^{M_{1}} \psi_{m}^{q_{\varepsilon}, q_{\eta}, q_{l}}\left(x^{\prime}, \mu\right) Y_{m, k, i, j}(\theta),
$$

where $\psi_{m}^{q_{\varepsilon}, q_{\eta}, q_{l}}$ is a linear combination of functions $u_{q_{\varepsilon}-1, q_{\eta}-1, q_{l}}$ and the derivatives of these functions, $M_{1}$ is some number depending on the superscripts of functions $\psi_{m}^{q_{\varepsilon}, q_{\eta}, q_{l}}$, and $Z_{m, k, i, j}$ are infinitely differentiable functions on the half-sphere $\overline{S_{1}^{n-1}}$ satisfying Neumann condition on its boundary. We shall check this statement later. In particular, functions $Z_{k, i, 0}^{1, q_{\eta}, 0}\left(x^{\prime}, \mu, \theta\right)$ satisfy the identity:

$$
Z_{k, i, 0}^{1, q_{\eta}, 0}\left(x^{\prime}, \mu, \theta\right)=\sum_{m=0}^{M_{1}} \psi_{m}^{1, q_{\eta}, 0}\left(x^{\prime}, \mu\right) Z_{m, k, i, 0}(\theta),
$$

where $\psi_{m}^{1, q_{\eta}, 0}$ is a linear combination of functions $u_{0, q_{\eta}-1,0}$ and the derivatives of these functions. This identity is implied by the asymptotics of function $w_{0, q_{\eta}-1,0}$ and right hand sides $G_{1, q_{\eta}, 0}$ at infinity.

We return back to constructing functions $w_{q_{\varepsilon}, q_{\eta}, q_{l}}$. Asymptotics of functions $w_{q_{\varepsilon}, q_{\eta}, q_{l}}$ at infinity should satisfy (4.20). As the result, we arrive at the identities:

$$
\begin{array}{ll}
\mathbf{B}^{q_{\varepsilon}, q_{\eta}, q_{l}}=\rho_{\varepsilon}\left(T_{0} \Psi_{q_{\varepsilon}, q_{\eta}, q_{l}}+A_{0}^{q_{\varepsilon}+1, q_{\eta}-1, q_{l}}\right), & Z_{2, i, j}^{q_{\varepsilon}, q_{\eta}, q_{l}}\left(x^{\prime}, \mu, \theta\right)=Y_{5, i, j}^{q_{\varepsilon}, q_{\eta}, q_{l}}\left(x^{\prime}, \mu, \theta\right), \\
Z_{1, i, j}^{q_{\varepsilon}, q_{\eta}, q_{l}}\left(x^{\prime}, \mu, \theta\right)=Y_{4, i, j}^{q_{\varepsilon}, q_{\eta}, q_{l}}\left(x^{\prime}, \mu, \theta\right), & Z_{3, i, j}^{q_{\varepsilon}, q_{\eta}, q_{l}}\left(x^{\prime}, \mu, \theta\right)=Y_{6, i, j}^{q_{\varepsilon}, q_{\eta}, q_{l}}\left(x^{\prime}, \mu, \theta\right) .
\end{array}
$$

The first identity in (4.26) is the solvability condition of the problems for functions $w_{q_{\varepsilon}, q_{\eta}, q_{l}}$. The validity of the second identity in (4.26) and the identity in (4.27) is proven in the same way as in Section 7 in [14. Identities (4.26), (4.27) determine coefficients $A_{i}^{q_{\varepsilon}, q_{\eta}, q_{l}}\left(x^{\prime}, \mu\right)$ and $C_{i}^{q_{\varepsilon}, q_{\eta}, q_{l}}\left(x^{\prime}, \mu\right)$ considered in (4.13) and (4.25), respectively. We recall that these coefficients and the solutions from Lemmata 4.1, 4.3 were added to functions $v_{q_{\varepsilon}, q_{\eta}, q_{l}}$ of boundary layer and to functions $w_{q_{\varepsilon}, q_{\eta}, q_{l}}$ of internal expansion, respectively. And each of these coefficients is a linear combination of all corresponding functions of the external expansion and their derivatives. This statement is implied by Remark 4.1 and 4.3, identities for the right hand sides in the definition of functions $G_{q_{\varepsilon}, q_{\eta}, q_{l}}$, the identities in (4.26), (4.27) and the definition of operator $\Delta_{\theta}^{N}$. The asymptotics of function $Y$ and identities (4.26), (4.27) explain the choice of solutions to the homogeneous problem in the boundary layer described above in Remark 4.2, By the first identity in (4.26) and by (2.12), (4.13), (4.14), (4.21) we obtained boundary conditions for the remaining terms $u_{q_{\varepsilon}, q_{\eta}, q_{l}}$ of external expansion:

$$
\begin{aligned}
\left(\frac{\partial}{\partial \nu}+a+\frac{N_{0}\left(\mu+K_{0}\right)}{T_{0}}\right) u_{q_{\varepsilon}, q_{\eta}, q_{l}}= & \frac{\mu+K_{0}}{T_{0}}\left(-N_{0}\left(\varrho \mathbf{A}^{q_{\varepsilon}, q_{\eta}, q_{l}}+\Psi_{q_{\varepsilon}-1, q_{\eta}, q_{l}} \widetilde{X}_{0}\right)\right. \\
& \left.+\bar{\delta}_{q_{\varepsilon}, 0} \mathbf{C}^{q_{\varepsilon}, q_{\eta}, q_{l}}\right)-\frac{A_{0}^{q_{\varepsilon}, q_{\eta}-1, q_{l}}}{T_{0}} \text { as } x \in \Upsilon .
\end{aligned}
$$

Here constant $\widetilde{X}_{0}$ and function $\varrho$ are defined in (4.11) and (4.21), respectively. It is clear that denominators in these identities are non-zero, since $T_{0}$ is non-zero (see the asymptotics of function $X$ ), while $\rho_{\varepsilon}$ tends to a constant as $\varepsilon \rightarrow 0$ by (2.10). Moreover, we mention that as $\left(q_{\varepsilon}, q_{\eta}, q_{l}\right)=(0,0,0)$, the right hand side in (4.28) vanishes due to the definition of functions $\varrho$, $\mathbf{A}^{q_{\varepsilon}, q_{\eta}, q_{l}}, \mathbf{C}^{q_{\varepsilon}, q_{\eta}, q_{l}}$ and $A_{0}^{q_{\varepsilon}, q_{\eta}, q_{l}}$. Relations (4.3), (4.4), (4.5) and (4.28) define the problems for coefficients $u_{q_{\varepsilon}, q_{\eta}, q_{l}}$.

Remark 4.4. While constructing the coefficients of internal expansion, we added some solutions to the homogeneous problem in Lemma 4.3 with arbitrary coefficients $C_{i}^{q_{\varepsilon}, q_{\eta}, q_{l}}\left(x^{\prime}, \mu\right)$. In 
accordance with Lemma 4.3, this problem has infinitely many solutions. At that, among the existing infinite set of solutions in this lemma, we added only the above mentioned ones. Adding of these terms is sufficient to satisfy (4.20) by the asymptotics of the constructed terms of the internal expansions. Thus, our apriori choice of the added solutions is due to the conditions, which appeared in matching with the coefficients of the internal expansion.

We denote $\Gamma_{3}:=\left\{\zeta: \zeta_{n} \geqslant 0, \zeta \notin \partial \Gamma_{1} \times\{0\}\right\}$. We choose $\delta>0$ such that the inclusion $\partial \Gamma_{1} \subset\left\{\zeta:\left|\zeta^{\prime}\right|<\delta, \zeta_{n}=0\right\}$ holds true. Here $\partial \Gamma_{1}$ is treated as the boundary of set $\Gamma_{1}$ of dimension $n-1$. By analogy with [14, Lm. 6.3] one can prove the next lemma.

Lemma 4.4. Problems (4.23) are solvable, functions $w_{q_{\varepsilon}, q_{\eta}, q_{l}}$ and $G_{q_{\varepsilon}, q_{\eta}, q_{l}}$ are represented as the sums

$$
\begin{aligned}
& w_{q_{\varepsilon}, q_{\eta}, q_{l}}\left(x^{\prime}, \mu, \zeta\right)=\sum_{q=1}^{M_{q_{\varepsilon}, q_{\eta}}^{1}} \rho_{q_{\varepsilon}, q_{\eta}, q_{l}}^{q}\left(x^{\prime}, \mu\right) w_{q_{\varepsilon}, q_{\eta}, q_{l}}^{q}(\zeta), \\
& G_{q_{\varepsilon}, q_{\eta}, q_{l}}\left(x^{\prime}, \mu, \zeta\right)=\sum_{q=1}^{M_{q_{\varepsilon}, q_{\eta}}^{2}} \phi_{q_{\varepsilon}, q_{\eta}, q_{l}}^{q}\left(x^{\prime}, \mu\right) G_{q_{\varepsilon}, q_{\eta}, q_{l}}^{q}(\zeta),
\end{aligned}
$$

where $M_{q_{\varepsilon}, q_{\eta}}^{i}$ are some numbers, $\rho_{q_{\varepsilon}, q_{\eta}, q_{l}}^{q}, \phi_{q_{\varepsilon}, q_{\eta}, q_{l}}^{q}$ are finite linear combinations of the traces of the coefficients of the external expansions and of their derivatives, $w_{q_{\varepsilon}, q_{\eta}, q_{l}}^{q} \in C^{\infty}\left(\Gamma_{3}\right)$, $w_{q_{\varepsilon}, q_{\eta}, q_{l}}^{q} \in W_{2}^{1}\left(\left\{\zeta: \zeta_{n}>0,|\zeta|<\delta\right\}\right), G_{q_{\varepsilon}, q_{\eta}, q_{l}}^{q} \in C^{\infty}\left(\Gamma_{3}\right), G_{q_{\varepsilon}, q_{\eta}, q_{l}}^{q} \in L_{2}\left(\left\{\zeta: \zeta_{n}>0,|\zeta|<\delta\right\}\right)$.

We denote $\Omega_{\tau_{0}-\delta}:=\left\{x: 0<\tau<\tau_{0}-\delta\right\}$.

Lemma 4.5. Let functions $f \in W_{2}^{m}\left(\Omega_{\tau_{0}}\right) \cap L_{2}(\Omega)$ and $\varphi \in W_{2}^{m+1}(\Upsilon)$ are holomorphic in $\mu$ in the sense of the norms in $W_{2}^{m}\left(\Omega_{\tau_{0}}\right) \cap L_{2}(\Omega)$ and $W_{2}^{m+1}(\Upsilon)$ for all $m \in \mathbb{N}$ and $u$ is the solution to the problem

$$
\begin{aligned}
&(\mathcal{L}-\lambda) u=f, \quad x \in \Omega \\
&\left(\frac{\partial}{\partial \nu}+a+\frac{N_{0}\left(\mu+K_{0}\right)}{T_{0}}\right) u=\varphi, \quad x \in \Upsilon, \quad\left(\frac{\partial}{\partial \nu}+a\right) u=0, \quad x \in \Xi .
\end{aligned}
$$

Then this problem is solvable in $W_{2}^{2}(\Omega)$, for each $m \in \mathbb{N}$ and each $\delta>0$ function $u$ belongs to $W_{2}^{m+2}\left(\Omega_{\tau_{0}-\delta}\right)$, is holomorphic w.r.t. $\mu$ in the sense of the norm in $W_{2}^{m+2}\left(\Omega_{\tau_{0}-\delta}\right) \cap W_{2}^{2}(\Omega)$ and the estimate

$$
\|u\|_{W_{2}^{m+2}\left(\Omega_{\tau_{0}-\delta}\right)} \leqslant C
$$

holds true, where constant $C$ is independent of $u, f, \mu$ and $\varphi$ but depends on $m$ and $\delta$.

Proof. The solvability of the problem for $\mu=0$ is implied by the assumption that $\lambda$ does not belong to the spectrum of operator $\mathcal{H}_{0}$. Therefore, there exists the inverse bounded operator $\left(\mathcal{H}_{0}-\lambda\right)^{-1}: L_{2}(\Omega) \rightarrow W_{2}^{2}(\Omega)$.

Let $\chi_{4}$ be an infinitely differentiable cut-off function vanishing as $x_{n}>\frac{\tau_{0}}{2}$ and being equal to one as $0<x_{n}<\frac{\tau_{0}}{3}$. We make the change of function $u$ :

$$
v(x, \mu)=u(x, \mu) \psi_{0}\left(x_{n}, \mu\right), \quad \psi_{0}\left(x_{n}, \mu\right):=1-\chi_{4}\left(x_{n}\right)\left(\mathrm{e}^{-C_{0} \mu x_{n}}-1\right), \quad C_{0}:=\frac{N_{0}}{T_{0}} .
$$

By (4.29), function $v$ is the solution to operator equation $\left(\mathcal{H}_{0}-\lambda-\mu L_{0}\right) v=F$, where $F:=$ $\psi_{0} f$, while $L_{0}$ is a bounded operator from $W_{2}^{1}(\Omega)$ into $L_{2}(\Omega)$ and has the form

$$
L_{0}:=\frac{\psi_{1}}{\psi_{0}}\left(\bar{A}_{n}-A_{n}-A_{n n}+\sum_{j=1}^{n} \frac{\partial A_{j n}}{\partial x_{j}}\right)+A_{n n} \frac{\psi_{2}}{\psi_{0}}+\frac{2 \psi_{1}}{\psi_{0}} \sum_{j=1}^{n} A_{n j} \frac{\partial}{\partial x_{j}},
$$


where

$$
\psi_{1}:=\frac{d \chi_{4}}{d x_{n}}+\mathrm{e}^{-C_{0} \mu x_{n}}\left(1-\chi_{4}\right), \quad \psi_{2}:=\frac{d^{2} \chi_{4}}{d x_{n}^{2}}-\mathrm{e}^{-C_{0} \mu x_{n}}\left(2 \frac{d \chi_{4}}{d x_{n}}+C_{0} \mu\left(1-\chi_{4}\right)\right) .
$$

We note that denominators in (4.30) are non-zero since function $\psi_{0}$ tends to a constant as $\mu \rightarrow 0$. It follows from the definition of operators $L_{0}$ and $\left(\mathcal{H}_{0}-\lambda\right)^{-1}$ that $\left(\mathcal{H}_{0}-\lambda\right)^{-1} L_{0}$ is a bounded operator in $L_{2}(\Omega)$. Hence, function $v$ is represented by the identity $v=\left(I-\mu\left(\mathcal{H}_{0}-\right.\right.$ $\left.\lambda)^{-1} L_{0}\right)^{-1}\left(\mathcal{H}_{0}-\lambda\right)^{-1} F$, which proves the solvability of the problem. Then expanding operator $\left(I-\mu\left(\mathcal{H}_{0}-\lambda\right)^{-1} L_{0}\right)^{-1}$ into Neumann series and using the definition of function $v$, we establish the holomorphy of function $u$ w.r.t. $\mu$ in the sense of norm in $W_{2}^{2}(\Omega)$.

By the smoothness of functions $f$ and $\varphi$, the holomorphy of these functions in $\mu$ and by the smoothness improving theorems [29, Ch. 4, Sect. 2] we establish that for each integer $m>0$ and each $\delta>0$, function $u$ belongs to space $W_{2}^{m+2}\left(\Omega_{\tau_{0}-\delta}\right)$ and satisfies the inequality

$$
\|u\|_{W_{2}^{m+2}\left(\Omega_{\tau_{0}-\delta}\right)} \leqslant C\left(\|f\|_{W_{2}^{m}\left(\Omega_{\tau_{0}}\right)}+\|u\|_{W_{2}^{1}(\Omega)}+\|\varphi\|_{W_{2}^{m+1}(\Upsilon)}\right) .
$$

Then by induction in $m$ one can prove easily the statement of the lemma on the holomorphy of function $u$ w.r.t. $\mu$ in the norm in $W_{2}^{m+2}\left(\Omega_{\tau_{0}-\delta}\right) \cap W_{2}^{2}(\Omega)$.

In accordance with the last lemma, the coefficients of the external expansion are holomorphic w.r.t. $\mu$. Together with the identities for the functions of the boundary layer and internal expansion it follows that these coefficients are also holomorphic w.r.t. $\mu$ in the norms in $W_{2}^{1}\left(\Pi^{\delta}\right)$ and $W_{2}^{1}\left(\zeta: \zeta_{n}>0,|\zeta|<\delta\right)$, respectively. By the previous lemma, functions $\varphi_{q_{\varepsilon}, q_{\eta}, q_{l}}^{q}$, $\psi_{q_{\varepsilon}, q_{\eta}, q_{l}}^{q}$ and $\rho_{q_{\varepsilon}, q_{\eta}, q_{l}}^{q}, \phi_{q_{\varepsilon}, q_{\eta}, q_{l}}^{q}$ considered in Lemmata 4.2 and 4.4 belong to $W_{2}^{m}(\Upsilon)$ for all $m \geqslant 0$. The formal construction of external expansion (2.16) and internal expansion (2.18) is complete. The justification reproduces that in [14, Sect. 7]. While justifying, various estimates for the norms of the coefficients of the boundary layer, external and internal expansions are obtained as well as the estimates for the error terms. At that, all the constants in these inequalities are uniformly bounded in $\varepsilon, \eta$ and $\mu$. This fact is implied by Lemma 4.5 and the explicit form of functions of boundary layer and internal expansion.

\section{Sharpness of EStimate}

In the present section we discuss the sharpness of the estimates established in Theorem 2.1. We shall show first the estimate (2.9) is true for a periodic structure of the alternation of boundary conditions. As an example we consider the hyperplane $\Upsilon:=\left\{x: x_{n}=0\right\}$ and we introduce set $\gamma_{\varepsilon}$. This set is introduced in the same way as in the previous section. Let $N:=\left[\frac{1}{\varepsilon}\right],[\cdot]$ be the integer part of a number, $b:=N \varepsilon, \square_{\varepsilon}^{i} \subset \Upsilon$ be a cube with side $\varepsilon$ such that $\gamma_{\varepsilon}^{(i)} \subset \square_{\varepsilon}^{i}$. In hyperplane $\Upsilon$ we choose a cube $Q$ with side $b$ containing an integer amount of sets $\square_{\varepsilon}^{i}$ such that the union of these cubes covers $\Upsilon$.

We denote $\beta_{\varepsilon}:=\alpha-\alpha_{\varepsilon}$. We expand function $\beta_{\varepsilon}$ into Fourier series $Q$ :

$$
\beta_{\varepsilon}\left(x^{\prime}\right):=\sum_{q \in \mathbb{Z}^{n-1}} C_{q} \mathrm{e}^{\frac{2 \pi \mathrm{i}}{b} q \cdot x^{\prime}} .
$$

We take the function $U(x)=-\sum_{q \in \mathbb{Z}^{n-1}} C_{q} \frac{\mathrm{e}^{\frac{2 \pi \mathrm{i}}{b} q \cdot x^{\prime}-(|q|+1) x_{n}}}{|q|+1}$. It is easy to check that the series converges in $W_{2}^{1}(\widetilde{\Sigma}), \widetilde{\Sigma}:=\left\{x: x_{n}>0, x^{\prime} \in Q\right\}$. Function $U$ is a generalized solution to the boundary value problem

$$
\Delta U=0, \quad x \in \widetilde{\Sigma}, \quad \frac{\partial U}{\partial x_{n}}=\beta_{\varepsilon}, \quad x^{\prime} \in Q
$$


with the homogeneous Neumann conditions on the lateral sides of $\widetilde{\Sigma}$.

In accordance with the definition of function $\beta_{\varepsilon}$, the identity

$$
\sum_{q \in \mathbb{Z}^{n-1}} \frac{1}{|q|+1}\left|\int_{Q} \beta_{\varepsilon} \mathrm{e}^{\frac{2 \pi \mathrm{i}}{b} q \cdot x^{\prime}} d x^{\prime}\right|^{2}=\sum_{q \in \mathbb{Z}^{n-1}} \frac{\left|C_{q}\right|^{2}}{|q|+1}
$$

holds true. On the other side, in accordance with the definition of function $U$, we have

$$
\|\nabla U\|_{L_{2}(\widetilde{\Sigma})}^{2}=2 \sum_{q \in \mathbb{Z}^{n-1}}\left|C_{q}\right|^{2} \int_{0}^{+\infty} \mathrm{e}^{-2(|q|+1) x_{n}} d x_{n}=C \sum_{q \in \mathbb{Z}^{n-1}} \frac{\left|C_{q}\right|^{2}}{|q|+1} .
$$

Therefore, we obtain

$$
\sum_{q \in \mathbb{Z}^{n-1}} \frac{1}{|q|+1}\left|\int_{Q} \beta_{\varepsilon} \mathrm{e}^{\frac{2 \pi \mathrm{i}}{b} q \cdot x^{\prime}} d x^{\prime}\right|^{2} \leqslant C\|\nabla U\|_{L_{2}(\widetilde{\Sigma})}^{2}
$$

Our next step is to estimate the right hand side of the last inequality. In order to do it, we introduce the following notations: $\square_{\varepsilon}:=\left\{x \in \mathbb{R}^{n-1}:-\frac{1}{2 N}<x_{j}<\frac{1}{2 N}\right.$, $\left.j=1, \ldots, n-1, x_{n}=0\right\}, \widetilde{\Sigma}_{1}:=\left\{x: x_{n}>0, x^{\prime} \in \square_{\varepsilon}\right\}, \xi=x \varepsilon^{-1}, \widetilde{\Sigma}_{\eta}:=\left\{\xi: \xi_{n}>0, \xi^{\prime} \in \square\right\}$, where $\square$ is the domain obtained by dilation of domain $\square_{\varepsilon}$ in $\varepsilon^{-1}$ times.

By analogy with [34], for $n \geqslant 3$ we can calculate the asymptotics for the lowest eigenvalue of Laplace operator in $\widetilde{\Sigma}_{\eta}$ subject to Neumann condition on $\square \backslash\{0\}$. The first non-zero term in the asymptotics is of order $\varepsilon$. Indeed, function $U$ satisfies

$$
\|U-\varepsilon u\|_{W_{2}^{1}\left(\widetilde{\Sigma}_{\eta}\right)}=O(\varepsilon \eta) .
$$

Here function $u$ is a solution to the problem

$$
\Delta_{\xi} u=0, \quad \xi \in \widetilde{\Sigma}_{\eta}, \quad \frac{\partial u}{\partial \xi_{n}}=0, \quad \xi \in \square \backslash\{0\},
$$

subject to periodic boundary conditions on the lateral sides of $\widetilde{\Sigma}_{\eta}$, having the asymptotics $u=|\xi|^{-n+2}+O\left(|\xi|^{-n+1}\right)$ as $|\xi| \rightarrow 0$.

In the same way as [14, Lm. 7.3], for $U \in W_{2}^{1}\left(\widetilde{\Sigma}_{\eta}\right)$ one can prove the estimate $\left\|\nabla_{\xi} U\right\|_{L_{2}\left(\widetilde{\Sigma}_{\eta}\right)}^{2} \leqslant$ $C \varepsilon^{\frac{3}{2}}$. Returning back to variables $x$ and taking into account that $\operatorname{set} Q$ contains $O\left(\varepsilon^{-n+1}\right)$ of periodicity cells, we obtain the estimate

$$
\|\nabla U\|_{L_{2}(\widetilde{\Sigma})}^{2} \leqslant C \varepsilon^{\frac{1}{2}}
$$

By (5.1) it follows that function $\kappa(\varepsilon)$ in (2.9) should be chosen as $C \varepsilon^{\frac{1}{4}}$ to satisfy this estimate for our example. It remains to check that function $\alpha$ vanishes on $\bar{\Upsilon} \cap \bar{\Xi}$ if this intersection is non-empty. In our case these sets are disjoint and we do not need to assume the vanishing of $\alpha$ on $\bar{\Upsilon} \cap \bar{\Xi}$. Thus, we have shown that for a periodic alternation there exists a function $\alpha$ and Condition (C2) is satisfied.

We proceed to discussing the sharpness of the estimates established in Theorem 2.1. If we try to prove the sharpness of estimate (2.13), it leads us to some rough estimates only. This is why we shall study only the sharpness of estimate (2.14).

In this section, by $C$ we denote inessential constants independent of the coefficients of external and internal expansions and boundary layer, as well as of $f, \varepsilon, \eta, \mu, \kappa(\varepsilon)$ and $x$. In addition we assume that $u_{0,0,0}$ and $f$ are compactly supported infinitely differentiable functions. We recall 
that the asymptotics of function $u_{\varepsilon}$ in the norm in $W_{2}^{1}(\Omega)$ is of the form (2.15). Then

$$
\begin{aligned}
\left\|u_{\varepsilon}-\left(1-W_{\varepsilon}\right) u_{0,0,0}\right\|_{W_{2}^{1}(\Omega)}= & \|\left(\varepsilon u_{1,0,0}+\eta u_{0,1,0}+\varepsilon \chi_{0} v_{1,0,0}+\ldots\right) \chi_{5} \\
& +\left(\chi_{5}-1+W_{\varepsilon}\right) u_{0,0,0}+\left(w_{0,0,0}+\ldots\right) \chi_{6} \|_{W_{2}^{1}(\Omega)}
\end{aligned}
$$

where by "..." we denote the next terms of the asymptotic series, function $W_{\varepsilon}$ is defined in (3.3), while functions $\chi_{5}, \chi_{6}$ are of the form:

$$
\chi_{5}:=\prod_{k \in \mathbb{Z}^{n-1}} \chi_{1}\left(\left|x-M_{\varepsilon}^{k}\right| \varepsilon^{-1} \eta^{-1 / 2}\right), \quad \chi_{6}:=\sum_{k \in \mathbb{Z}^{n-1}}\left(1-\chi_{1}\left(\left|x-M_{\varepsilon}^{k}\right| \varepsilon^{-1} \eta^{-1 / 2}\right)\right) .
$$

Let us estimate the norm of $\chi_{6} w_{0,0,0}+\varepsilon \chi_{0} \chi_{5} v_{1,0,0}+u_{0,0,0} W_{\varepsilon}$ in $W_{2}^{1}(\Omega)$. In accordance with the definition of functions $\chi_{0}, \chi_{1}, \chi_{2}, w_{0,0,0} v_{1,0,0}, W_{\varepsilon}$ and the properties of function $u_{0,0,0}$ we obtain

$$
\begin{aligned}
& \left\|\chi_{6} w_{0,0,0}+\varepsilon \chi_{0} \chi_{5} v_{1,0,0}+u_{0,0,0} W_{\varepsilon}\right\|_{W_{2}^{1}(\Omega)}^{2} \geqslant C_{1} \sum_{i=1}^{N(\varepsilon)}\left\|\frac{\partial w_{0,0,0}}{\partial x_{n}}+u_{0,0,0} \frac{\partial W_{\varepsilon}}{\partial x_{n}}\right\|_{L_{2}\left(B^{k, 1}\right)}^{2} \\
& +C_{2} \sum_{i=1}^{N(\varepsilon)}\left\|\varepsilon \frac{\partial v_{1,0,0}}{\partial x_{n}}+u_{0,0,0} \frac{\partial W_{\varepsilon}}{\partial x_{n}}\right\|_{L_{2}\left(B^{k, 2}\right)}^{2}=C_{1}(\varepsilon \eta)^{-2} \sum_{i=1}^{N(\varepsilon)}\left\|-T^{0,0,0} \frac{\partial Y}{\partial \zeta_{n}}+u_{0,0,0} \frac{\partial Y}{\partial \zeta_{n}}\right\|_{L_{2}\left(B^{k, 1}\right)}^{2} \\
& +C_{2} \varepsilon \sum_{i=1}^{N(\varepsilon)}\left\|\Psi_{0,0,0} \frac{\partial X}{\partial \xi_{n}}+u_{0,0,0} \frac{\partial W_{\varepsilon}}{\partial \xi_{n}}\right\|_{L_{2}\left(B^{k, 2}\right)}^{2}
\end{aligned}
$$

where sets $B^{k, 1}$ and $B^{k, 2}$ are of the form $B^{k, 1}:=\left\{x:\left|x-M_{\varepsilon}^{k}\right| \varepsilon^{-1} \eta^{-1 / 2}<1\right\}$, $B^{k, 2}:=\left\{x:\left|x-M_{\varepsilon}^{k}\right| \varepsilon^{-1} \eta^{-1 / 2}>1\right\} \cap \Omega_{\tau_{0}}$. The last identity, (4.28), asymptotics of functions $X$ in a small neighbourhood of zero and of $Y$ at infinity, as well as the definitions of functions $T^{0,0,0}, W_{\varepsilon}$ imply

$$
\left\|\chi_{6} w_{0,0,0}+\varepsilon \chi_{0} \chi_{5} v_{1,0,0}+u_{0,0,0} W_{\varepsilon}\right\|_{W_{2}^{1}(\Omega)} \geqslant C \varepsilon^{\frac{1}{2}} .
$$

By (5.2), Lemma 7.3 in [14] and the definition of function $W_{\varepsilon}$ it follows that

$$
\left\|u_{\varepsilon}-\left(1-W_{\varepsilon}\right) u_{0,0,0}\right\|_{W_{2}^{1}(\Omega)} \geqslant C \varepsilon^{\frac{1}{2}}
$$

This estimate differs from the right hand side of inequality (2.14). Namely, the first term coincides with the similar one in the estimate for the convergence rate. It means that this term is sharp. The third term, $\kappa(\varepsilon)$, in our estimate comes from Lemma 3.5. And as it was said, the proof of this lemma is based on sharp estimates. It gives us an opportunity to assume that term $\kappa(\varepsilon)$ in inequality (2.14) is also sharp.

Let us study the sharpness of term $\mu$ in the right hand side of (2.14). Since the coefficients of the boundary layer, external and internal expansions are holomorphic in $\mu$, as well as by the fact that we do not construct asymptotics of function $u_{\varepsilon}$ in powers of $\mu$, we obtain an error of order $\mu$. Hence, by inequality (5.3), Lemma 7.3 in [14] we obtain that

$$
\left\|u_{\varepsilon}-\left(1-W_{\varepsilon}\right) u_{0,0,0}\right\|_{W_{2}^{1}(\Omega)} \geqslant C\left(\varepsilon^{\frac{1}{2}}+\mu\right) .
$$

Thus, our estimate (2.14) is close to being sharp. 


\section{BIBLIOGRAPHY}

1. G.A. Chechkin. Averaging of boundary value problems with singular perturbation of the boundary conditions // Matem. Sborn. 184:6, 99-150. [Russ. Acad. Sci. Sb. Math. 79:1, 191-220 (1994).]

2. R.R. Gadyl'shin. Homogenization and asymptotics for a membrane with closely spaced clamping points // Zhurn. Vychisl. Mat. Mat. Fiz. 41:12, 1857-1869 (2001). [Comput. Math. Math. Phys. 41:12, 1765-1776 (2001).]

3. A. Friedman, Ch. Huang, J. Yong. Effective permeability of the boundary of a domain // Commun. Part. Diff. Equat. 20:1-2, 59-102 (1995).

4. G.A. Chechkin. On boundary-value problems for second-order elliptic equations with oscillating boundary conditions // Non-classical partial differential equations, Inst. Math. Sibirsk. Otdel. Akad. Nauk SSSR, Novosibirsk 95-104 (1988). (in Russian)

5. A. Damlamian, Li Ta-Tsicn (Li Daqian). Boundary homogenization for ellpitic problems // J. Math. Pure et Appl. 66:4, 351-361 (1987).

6. M. Lobo, E. Perez. Asymptotic behaviour of an elastic bodx with a surface having small stuck regions // RAIRO Model. Math. Anal. Numer. 22:4, 609-624 (1988).

7. G. Chechkin, E. Doronina. On the asymptotics of the spectrum of a boundary value problem with nonperiodic rapidly alternating boundary conditions // Funct. Differ. Equ. 8:1-2, 111-122 (2001).

8. O. Oleinik, G. Chechkin. Solutions and eigenvalues of the boundary value problems with rapidly alternating boundary conditions for the system of elasticity // Rendic. Linc. Matem. Applic. Scric IX. 7:1, 5-15 (1996).

9. D.I. Borisov. Asymptotics and estimates for the eigenelements of the Laplacian with frequently alternating nonperiodic boundary conditions // Izv. RAN. Ser. Matem. 67:6, 23-70 (2003). [Izv. Math. 67:6, 1101-1148 (2003).]

10. D.I. Borisov. On a problem with nonperiodic frequent alternation of boundary condition imposed on fast oscillating sets // Zhurn. Vychisl. Matem. Matem. Fiz. 46:2, 284-294 (2006). [Comp. Math. Math. Phys. 46:2, 271-281 (2006).]

11. E.I. Doronina, G.A. Chechkin. On the averaging of solutions of a second order elliptic equation with nonperiodic rapidly changing boundary conditions // Vestn. Mosk. Univ. Ser. I. 1, 14-19 (2001). [Mosc. Univ. Math. Bull. 56:1, 14-19 (2001).]

12. A.G. Belyaev, G.A. Chechkin. Homogenization of a mixed boundary-value problem for the Laplace operator in the case of an insoluble 'limit' problem // Matem. Sborn. 186:4, 47-60 (1995). [Sb. Math. 186:4, 511-525 (1995).]

13. T.F. Sharapov. On the resolvent of multidimensional operators with frequently changing boundary conditions in the case of the homogenized Dirichlet condition // Matem. Sborn. 205:10, 125-160 (2014). [Sb. Math. 205:10, 1492-1527 (2014).]

14. D.I. Borisov, T.F. Sharapov. On resolvent on multi-dimensional operators with frequent alternation of boundary condition in the case of Robin homogenized boundary condition // Probl. Matem. Anal. 83, 3-40 (2015). [J. Math. Sci. 213:4, 3-40 (2016).]

15. D. Borisov, G. Cardone. Homogenization of the planar waveguide with frequently alternating boundary conditions // J. Phys. A. 42:36, id 365205 (21pp) (2009).

16. D. Borisov, R. Bunoiu, G. Cardone. On a waveguide with frequently alternating boundary conditions: homogenized Neumann condition // Ann. H. Poincaré. 11:8, 1591-1627 (2010).

17. D. Borisov, R. Bunoiu, G. Cardone. Homogenization and asymptotics for a waveguide with an infinite number of closely located small windows // J. Math. Sci. 176:6, 774-785 (2011).

18. D. Borisov, R. Bunoiu, G. Cardone. Waveguide with non-periodically alternating Dirichlet and Robin conditions: homogenization and asymptotics // Z. Angew. Math. Phys. 64:3, 439-472 (2013).

19. D. Borisov, G. Cardone, T. Durante. Norm resolvent convergence for elliptic operators in domain with perforation along curve // C.R. Math. 352:9, 679-683 (2014).

20. D. Borisov, G. Cardone, T. Durante. Homogenization and norm resolvent convergence for elliptic operators in a strip perforated along a curve // Proc. Royal Soc. Edinburgh, Sec. A. Math., To appear. 
21. A.G. Belyaev, A.L. Piatnitski, G.A. Chechkin. Asymptotic behavior of the solution of a boundary value problem in a punctured domain with an oscillating boundary // Sibir. Matem. Zhurn. 39:4, 730-754 (1998). [Siber. Math. J. 39:4, 621-644 (1998).]

22. A.G. Belyaev, A.L. Piatnitski, G.A. Chechkin. Averaging in a perforated domain with an oscillating third boundary condition // Matem. Sborn. 192:7, 3-20 (2001). [Sb. Math. 192:7, 933-949 (2001).]

23. R.R. Gadyl'shin. The asymptotics of eigenvalues of the boundary value problem with rapidly oscillating boundary conditions // Differ. Uravn. 35:4, 540-551 (1999). [Diff. Equat. 35:4, 540-551 (1999).]

24. D.I. Borisov, R.R. Gadyl'shin. On the spectrum of the Laplacian with frequently alternating boundary conditions // Teor. Mat. Fiz. 118:3, 347-353. [Theor. Math. Phys. 118:3, 272-277 (1999).]

25. G. A. Chechkin. The asymptotic expansion of the solution of a boundary-value problem with rapidly alternating type of boundary conditions // Trudy Semin. im. I. G. Petrovskogo 19, 323-337 (1996). [J. Math. Sci. 85:6, 2440-2449 (1997).

26. D. Borisov. On a model boundary value problem for Laplacian with frequently alternating type of boundary condition // Asympt. Anal. 35:1, 1-26 (2003).

27. D.I. Borisov. Boundary value problem in a cylinder with frequently changing type of boundary condition // Matem. Sborn. 193:7, 37-68 (2002). [Sb. Math. 193:7, 977-1008 (2002).]

28. T. Kato. Perturbation theory of linear operators. Springer-Verlag, Berlin (1996).

29. V.P. Mikhajlov. Partial differential equations. Nauka, Moscow (1976). [Mir Publishers, Moscow (1978).]

30. M.I. Višik and L.A. Ljusternik. Regular degeneration and boundary layer for linear differential equationswith small parameter // Uspekhi Mat. Nauk. 12:5, 3-122 (1957). [Amer. Math. Soc. Transl. 20:2, 239-364 (1962).]

31. N.N. Bogolyubov and Yu.A. Mitropol'skii. Asymptotic methods in the theory of non-linear oscillations Nauka, Moscow (1974). [Gordon and Breach, New York (1961).]

32. A.M. Il'in. Matching of asymptotic expansions of solutions of boundary value problems. Nauka, Moscow (1989). [Transl. Math. Monog. 102. Amer. Math. Soc., Providence, RI (1992).]

33. A.F. Nikiforov, S.K. Suslov, V.B. Uvarov. Classical orthogonal polynomials of a discrete variable. Nauka, Moscow (1985). [Springer Ser. Comp. Phys. Springer, Berlin (1991).]

34. R.R. Gadyl'shin. Asymptotic properties of an eigenvalue of a problem for a singularly perturbed self-adjoint elliptic equation with a small parameter in the boundary conditions // Differ. Uravn. 22:4, 640-652 (1986). [Differ. Equat. 22:4, 474-483 (1986).]

Timur Farkhatovich Sharapov,

Bashkir State Pedagogical University named after M. Akhmulla,

October rev. str 3a,

450000, Ufa, Russia

E-mail: stf0804@mail.ru 